
\title{
3 Research Square \\ Classification and Clinical Value of Three Immune Subtypes of Ovarian Cancer Based on Transcriptome Data
}

\section{Li Yuan (D229158546@qq.com )}

Zunyi Medical University

\section{Qiang An}

Zunyi Medical University

Ting Liu

Zunyi Medical University

Jukun Song

Guizhou provinicial people's hospital

\section{Research Article}

Keywords: Ovarian Cancer, Tumor-Infiltrating Immune Cells, Single-Sample Gene Set Enrichment Analysis

Posted Date: February 8th, 2021

DOl: https://doi.org/10.21203/rs.3.rs-163706/v1

License: (c) This work is licensed under a Creative Commons Attribution 4.0 International License. Read Full License

Version of Record: A version of this preprint was published at All Life on January 1st, 2021. See the published version at https://doi.org/10.1080/26895293.2021.1987339. 
Classification and clinical value of three immune subtypes of ovarian cancer based on transcriptome data

Li Yuan ${ }^{1}$, Qiang An ${ }^{1}$, Ting Liu ${ }^{1}$, Jukun Song ${ }^{2}$

1. Department of Gynecology, Affiliated Hospital of Zunyi Medical University, Guizhou, China.

2. Department of Oral and Maxillofacial Surgery, Guizhou Provincial People's Hospital, Guizhou, China.

\# Correspondence should be addressed to:

Li Yuan

Department of Gynecology, Affiliated Hospital of Zunyi Medical University, Guizhou, China.

Tel.: (+86) 0851-85922979

Fax: $(+86)$ 0851- 85924943

Email:rzent@163.com 


\title{
Classification and clinical value of three immune subtypes of ovarian cancer based on transcriptome data
}

\begin{abstract}
:
Background: Abundant evidence suggests that tumor immune infiltration was involved in the occurrence of ovarian cancer (OvCa). Current studies have demonstrated the effect of tumor-infiltrating immune cells (TIICs) on OvCa development; few studies have found the immune genomic profile and immune subclasses of OvCa based on transcriptome data, which may help to optimally stratify patients who respond to immunotherapy.
\end{abstract}

Methods: Based on the two publicly available OvCa transcriptomics data, three immunogenomic subgroups were classified using unsupervised hierarchical clustering. The GO and KEGG were analyzed in each subtype. Response to immunotherapy and anti-cancer drug targets was predicted by the TIDE, Submap algorithm, and GDSC dataset.

Results: The three types of immunogenomic OvCa subsets were classified based on immune signatures. We identified three OvCa subtypes, termed hyperimmunogenic (Immunity_H), moderately immunogenic (Immunity_M), and hypoimmunogenic (Immunity_L). Each subtype has specific pathways. In the Immunity_H subtype, a number of cancer-related and immune-related pathways are overactivated. In contrast, the Immunity_Lsubtype is predominantly enriched in lipid metabolism. Immunity_H subtype has higher immune cell infiltration, antitumor immunoreactivity, and better survival prognosis compared to other subtypes. Predicted clinical response to immune checkpoint blockade was used by Submap and TIDE algorithm and screened potential chemotherapeutic drug targets for OvCa was employed using GDSC. After the prediction for potential drug targets, we identified several potential drug targets for the treatment of OvCa, including Parthenolide and AKT inhibitor VIII, Paclitaxel. Also, 
Immunity_H subgroup has an early FIGO stage, and was susceptible to respond to immunotherapy.

Conclusions: The characterization of immune-based OvCa subgroups possessed potential clinical implications for $\mathrm{OvCa}$ treatment and has the potential to guide personalized treatment of OvCa patients through immunotherapy.

Keywords: Ovarian Cancer; Tumor-Infiltrating Immune Cells; Single-Sample Gene Set Enrichment Analysis

\section{Introduction}

As the second most common type of cancer in women, Ovarian cancer (OvCa) has a high risk of leading mortality worldwide. OvCa has an estimated incidence of 22,530 new cases and 13,980 estimated deaths in $2019^{1,2}$. Considering that the ovaries are located in the pelvic cavity, the early symptoms are not obvious, and about half of the patients are diagnosed in the late stage. Therefore, OvCa has the worst prognosis among all gynecological tumors. In most countries, the 5-year survival rate is still less than $45 \%^{3}$. Despite significant advances in surgery and chemotherapy, there is still a considerable gap to improve the prognosis. Many studies have found OvCa to be an immunogenic disease, so it was important to understand how immune cells was involved in the tumor microenvironment during tumor development ${ }^{4-6}$.

Tumor inflammation exerts a vital role in the occurrence and development of cancer. Multiple studies have demonstrated that tumor-infiltrating immune cells (TIIC) can help the host resist cancer cells and promote the development of solid tumors ${ }^{7-9}$, especially in the $\mathrm{OvCa}^{10,11}$. Recently, Immunotherapy promises to be a promising option for cancer treatment, and it can also prevent drug resistance. For some types of cancer (e.g. malignant melanoma), it has yielded satisfactory results but has not worked well for $\mathrm{OvCa}{ }^{12,13}$. Therefore, It's worth considering immunotherapy in $\mathrm{OvCa}$, as the treatment options for this disease are very limited. However, at present, Immunotherapy 
has shown beneficial effects in less than 20 percent of cancer patients. This suggests that not all OvCa patients respond to immunotherapy ${ }^{14,15}$. In the present work, Three immunogenomic OvCa subtypes were identified based on immune signatures in two publicly datasets. We have also identified OvCa subtypes that are sensitive to immunotherapy, which will help to explore the reasons for the poor response of $\mathrm{OvCa}$ to immunotherapy.

\section{Materials and Methods}

\section{Data source and processing}

RNA-seq data were acquired from two publicly available datasets: The Cancer Genome Atlas (TCGA) and the International Cancer Genome Consortium (ICGC) cohorts. The TCGA dataset included 380 primary tumor patients, ICGC dataset enrolled 85 patients. We simultaneously obtained the OvCa mutation data from the TCGA website. The clinical data was also downloaded.

\section{Single-Sample Gene Set Enrichment Analysis (ssGSEA) and Unsupervised clustering analysis}

Firstly, the single-sample gene set enrichment analysis (ssGSEA) was used to quantify the enrichment level based on the 29 immune signals in each OvCa sample ${ }^{16}$, 17. Secondly, the unsupervised hierarchical clustering among OvCa samples was performed based on the immune enrichment scores(Supplementary Excel).

Estimation of immune cell infiltration level, tumor purity, and stromal content in OvCa

The ESTIMATE method ${ }^{18}$ was employed to estimate the level of immune cell infiltration including immune score, tumor purity, and stromal score for each $\mathrm{OvCa}$ sample. We used the Kruskal-Wallis method to examine the difference between OvCa subtypes.

\section{Survival analysis}

We compared the survival analysis of three OvCa subsets in the ICGC and TCGA 
datasets separately. Kaplan-Meier curves were drawn to exhibit differences in survival rate. Log-rank tests were employed to examine the significance of survival differences. A P value less than 0.05 was considered statistically significant.

\section{The proportion of immune cell subpopulations in OvCa subtypes}

We used the CIBERSORT algorithm ${ }^{19}$ for inferring the proportion of LM22 human immune cells in TCGA and ICGC. We used 1000 permutations and $\mathrm{P}<0.05$ as inclusion criteria for tumor samples. The LM22 human cells among the three subclasses were compared. The Kruskal-Wallis test was used to examine the difference among OvCa subtypes.

\section{Gene Set Enrichment Analysis}

The gene enrichment analysis (GSEA) was employed to calculate the overall enrichment score in the TCGA data set ${ }^{20}$. The signature gene sets curated from $\mathrm{c} 2 / \mathrm{c} 5$ was downloaded from Broad Institute's Molecular Signature Database (MSigDB). Then we compared the KEGG pathway between Immunity_H and Immunity_L. According to a false discovery rate (FDR) $<0.05$, a significant enrichment pathway was identified.

\section{Mutation analysis}

We downloaded the mutation profiling in MAF of OvCa samples in the TCGA data set. We performed the mutation analysis using the $\mathrm{R}$ package "maftools"21, which calculate the mutation frequency of given genes and compare the most mutant genes between Immunity_H and Immunity_L. A P value $<0.05$ was considered statistically significant.

\section{Prediction of immunotherapy response and anti-cancer drug target}

We predicted the immunotherapy response using the tumor immune dysfunction and rejection (TIDE) algorithm ${ }^{22}$ and subclass mapping ${ }^{23}$. We also used the public pharmacogenomics database [Genomics of Drug Sensitivity in Cancer (GDSC) https://www.cancerrxgene.org/] for predicting drug response in subtypes of samples using the R package of pRRophetic ${ }^{24}$. Half-peak inhibition concentration (IC50) and 
prediction accuracy were demonstrated by ridge regression and 10-fold cross-validation depending on the GDSC training set, respectively.

\section{Statistical analysis}

All statistical tests were conducted using R software version 4.0.0. The Wilcox test was used to compare two groups, while the Kruskal-Wallis test was used for more than two groups ${ }^{25}$. Kaplan-Meier curve was performed to identify prognostic related subtypes for survival data ${ }^{26}$. Statistically significant differences were assessed by the log-rank test using the R package "survival". All $\mathrm{P}$ values were set as bilateral, and a Pvalue $<0.05$ was regarded as statistically significant.

\section{Results}

\section{Identification of Immunogenomic OvCa subtypes}

Firstly, a set of 29 immune-related genes that represent multiple immune cell types, functions, and pathways was downloaded. Secondly, we used the ssGSEA method to estimate the enrichment score of immune cells in tumor samples. Thirdly, immunogenomic OvCa subtypes were identified by unsupervised cluster analysis in the OvCa dataset. The two datasets exhibited similar clustering results, with three clusters separated. The three clusters: hyperimmunogenic (Immunity_H), moderately immunogenic (Immunity_M), and hypoimmunogenic (Immunity_L) were observed (Figure 1A, B).

In all data sets, stroma scores and the immune scores in the Immunity_H subset were significantly higher, while the tumor purity in the Immunity_L subset was significantly higher (Kruskal-Wallis test, $\mathrm{P}<0.05$ ) (Figure 2A, B). These findings indicated that the Immunity_H subtype had a higher percentage of immune and stromal cells, while the Immunity_L subtype contained a higher percentage of tumor cells.

Survival analysis results demonstrated that these OvCa subtypes have different clinical outcomes. The Immunity_H exhibited a better survival prognosis than the Immunity_M and Immunity_L in both datasets, but no statistically significant difference between the Immunity_M and Immunity_L subtypes in the ICGC dataset was detected (Figure 3A, B). 


\section{Comparisons of HLA and immune checkpoint among three OvCa}

\section{subtypes}

In thymic T-cell development, T-cell responses to autoantigens are eliminated, while T-cells, for exogenous antigenic responses by their human leukocyte antigenpresenting (HLA) molecules, are preserved. The HLA locus is located on chromosome 6 and covers a $7.6 \mathrm{Mb}$ region that contains more than 250 highly polymorphic genes. The region is divided into three subregions: class I, II, and III, all of which have distinct functions. It is reported that the HLA complex is one of the important components of the immune system ${ }^{27,28}$. In the current study, HLA-associated genes showed significantly higher expression in Immunity_H and lower expression in Immunity_L (Kruskal-Wallis test, $\mathrm{P}<0.05$, Figure 4A, B).

The expression levels of various immune cell marker genes such as IL3RA (pDC), IL-17 (Th17 cells), FOXP3 (Treg), CXCR5 (Tfh cells), CD1A (iDC), and CD8A (cytotoxic T cells) ${ }^{29}$ showed the same trend, which was highest in Immunity_H and lowest in Immunity_L. These findings also proved that Immunity_H obtains a higher concentration of immune cell infiltration.

We analyzed gene expression of PD-L1 (programmed death-ligand 1), PD1 (benzoate dehydratase 1), and PD-L2 (programmed death-ligand 2) for three $\mathrm{OvCa}$ subtypes in two datasets. The findings demonstrated that Immunity_H exhibited the highest expression of PD-L1, PD1, and PD-L2, while the lowest expression of PD-L1, PD1, and PD-L2 was observed in the Immunity_L (Kruskal-Wallis test, P $<0.05$ ) (Figure 5A, B). Based on the above results, we speculate that the OvCa subtype Immunity_H may respond better to PD-L1 immunotherapy because PD-1 / PD-L1 expression is usually positively correlated with more or less in vivo immune response ${ }^{30}$. Identification of subtype-specific immune cell infiltration patterns among the three OvCa subsets

The CIBERSOFT method using gene expression profiling was applied to impute the proportion of LM22 infiltration in OvCa samples. The results demonstrated that 
significant differences between OvCa subtypes in 11 of 22 immune cells; In the Immunity_H subgroup, activated dendritic cells, macrophage M1, T-cell CD8, T-cell follicular helper cells, and T-cell CD4 memory were activated, whereas dendritic cells were activated and monocytes were significantly reduced in the subgroup Immunity_L of the TCGA dataset (Figure 6A). In the ICGC dataset, differences between OvCa subtypes were observed for only 5 of the 22 immune cells. Dendritic cells activated, plasma cells in the Immunity_H subgroup were elevated, while in the Immunity_L subset monocyte and T-cell regulatory (Treg) was reduced (Figure 6B). This result is lined with previous results that the Immunity_H subtype was rich in immune cells.

\section{clinical characteristics of OvCa subtypes}

In terms of clinical features, Immunity_H had a young population (Age less than 65) and early FIGO TNM stage compared to Immunity_L in the TCGA data set. Immunity_L had advanced the FIGO TNM stage (Figure 7A, B, C). In the ICGC data set, we found similar results that Immunity_H had a young population (Age less than 65) and early FIGO TNM stage compared to Immunity_L. During the follow-up, Immunity_H had a higher complete remission, while Immunity_L had a higher relapse. The patients in the Immunity_L subset were mainly FIGO stage II, while patients with FIGO stage IV were enriched in the Immunity_H subset (Figure 7D, E, F).

\section{Identification of subtype-specific GO and KEGG Pathway}

In the TCGA dataset, GSEA analysis reveals subtype-specific GO and KEGG Pathway categories between Immunity_H and Immunity_L subtype (Figure 8A, B). Several immune-associated GO categories were enriched in the Immunity_H subtype, including MHC class II protein complexes, regulation of natural killer cell chemotaxis, and neutrophil activation. Compared with the Immunity_H subtype, iron-sulfur cluster assembly, and intestinal lipid absorption, triglyceride lipase activity was activated in the Immunity_L subset. Similarly, the immune-associated KEGG pathways were hyperactivated in the Immunity_H subclass and included Th17 cell differentiation, B cell receptor signaling pathway, $\mathrm{PD}-1$ checkpoint pathway in cancer, and $\mathrm{B}$ cell and $\mathrm{T}$ cell receptor signaling pathways. These findings reconfirmed that immunoreactivity 
was elevated in the subtype Immunity_H. In addition, several cancer-related pathways are overactivated in the Immunity_H subtype, such as the JAK-STAT signaling pathway, the NOD-like receptor signaling pathway, the TNF signaling pathway, and the Toll-like receptor signaling pathway. In contrast, the Immunity_L subtype is predominantly associated with enrichment in pathways related to porphyrin and chlorophyll metabolism, protein digestion, and insulin secretion.

\section{Comparison of OvCa subtype-specific gene mutations}

The OvCa subtype-specific gene mutations were explored between the Immunity_H and Immunity_Lsubtype. Highly mutated genetic profiles were exhibited in Figure 9A, B. The TP53, TTN, and CSMD3 were the most frequently mutated genes in the Immunity_H subtype, while TP53, TTN, and RYR2 were observed in the Immunity_Lsubtype. The USH2A and SYNE2 showed high mutation frequency in the Immunity_Lsubtype, with cutoffs less than 0.05 (Figure 9C).

\section{Prediction for Immunotherapy and Anti-cancer Drug Response}

Immunotherapy utilizing monoclonal antibodies against the immune checkpoint inhibitor(ICPC) shows a potential application for OvCa. Immunity_H responded better to treatment with ICPC compared with Immunity_Laccording to the TIDE algorithm $(\mathrm{P}<0.05)$. The expression profiles of OvCa subtypes were compared with another published data set using the Submap algorithm for the specific immune checkpoint. The findings suggested that the patients in Immunity_H were highly sensitive to programmed cell death 1 (PD1) immunotherapy (Bonferroni correction $\mathrm{P}<0.05$ ) (Figure 10A).

Anti-tumor drug therapy is one of the basic treatments for patients with OvCa. The prediction model was employed to assess the response to anti-cancer drugs between two molecular subgroups based on the GDSC cell line dataset. The prediction accuracy of the approach was confirmed with 10-fold cross-validation, and the response sensitivity was estimated with IC50. Among the 138 types of anti-cancer drugs, 85 exhibited the difference between Immunity_H and Immunity_L subtype (Supplementary Table 2). The result suggested that Immunity_H was more sensitive to 
these kinds of drugs than Immunity_L. Parthenolide and AKT inhibitor VIII, Paclitaxel exhibit great potential treatment value for $\mathrm{OvCa}(\mathrm{P}<0.05)$ (Figure 10B). In particular, paclitaxel as the first-line chemotherapy has been widely used in clinical practice.

\section{Discussion}

Since OvCa remains the worst prognosis among gynecological tumors, it is necessary to find new therapeutic targets to improve the prognosis of $\mathrm{OvCa}$ and make more efforts in this field to reveal the role of immune infiltration in $\mathrm{OvCa}^{11,31,32}$. Normally, immune cells recognize and attack cancer cells to inhibit their growth. However, cancer cells have an immune escape mechanism, which makes tumor initiation and progression extremely complex. The above-mentioned research results indicate that the tumor microenvironment, especially immune cells, exerts a vital role in the occurrence and development of ovarian cancer.

More and more pieces of evidence show that tumor immune infiltration is related to the prognosis of ovarian cancer ${ }^{33,34}$. The tumor immune microenvironment is a complex immune system, and further understanding of the mechanisms of TIIC in tumors may lead to the discovery of prognostic biomarkers and new targets for cancer therapy. Recently, Tumor-related macrophages (TAMs) were recognized as the most abundant immune stromal cells in the tumor microenvironment and an important regulator of tumor progression ${ }^{35}$. Macrophages have long been considered to be great plasticity in their phenotype and function which can be polarized into two mainstreams, classically activated macrophage (M1) and activated macrophage (M2) ${ }^{36}$. M1 macrophages could exert a pro-inflammatory function and boost the Th1 response and were inclined to identify and arrest tumor cells, therefore they emerged as the most prognostic immune cell type in ovarian cancer ${ }^{37,38}$. The subsets of memory $\mathrm{T}$ cells include $\mathrm{CD}^{+}$and $\mathrm{CD} 8^{+}$memory $\mathrm{T}$ cells. $\mathrm{CD}^{+}$memory $\mathrm{T}$ cells can eliminate tumor cells through secondary recognition of tumor-associated antigen. In the meantime, $\mathrm{CD}^{+}$memory $\mathrm{T}$ cells inhibit the growth of tumor cells by promoting the proliferation of $\mathrm{CD}^{+}$memory $\mathrm{T}$ cells ${ }^{39}$. Increasing studies suggested that T cells CD4 memory activated are associated with improved prognoses, such as non-small cell lung cancer, 
pancreatic adenocarcinomas, breast cancer, and cervical cancer ${ }^{40}$.

The traditional morphology-based classification system of ovarian cancer include histopathological classification (serous (SC), endometrioid (EC), clear cell (CCC), and mucinous (MC) carcinomas). OvCa can also be classified into type I (low grade) and type II (high grade) based on histology, pathogenesis, tumor growth, prognosis, and response to treatment ${ }^{41}$. Meanwhile, Previous studies have identified four phenotypebased expression-based high-grade serous tuboovarian cancer (HGSOC) molecular subtypes based on the transcriptome data, including $\mathrm{C} 1 /$ mesenchymal (C1.MES) subtype, C2/immunoreactive (C2.IMM) subtype, C4/Differentiated (C4.DIF) subtype, and $\mathrm{C} 5 /$ Proliferative (C5.PRO) subtype ${ }^{42,43}$. Currently, there is a lack of reliable molecular prognostic biomarkers for laryngeal cancer stratification based on DNA methylation profiles. Therefore, we identified subgroups of immune-related molecules based on the transcriptome data and evaluated the specific mechanisms in each subgroup in the work. In the work, the immunogenomic OvCa subsets were classified based on immune signatures using transcriptome data. Our findings show that $\mathrm{OvCa}$ can be divided into three subsets: Immunity_H, Immunity_M, and Immunity_L. Each subtype has specific characteristics. For example, Immunity_H had higher immune cell infiltration and stromal score, and a better prognosis, while Immunity_L contains higher tumor purity. Immunity_H has an early FIGO stage, while Immunity_L has an advanced FIGO stage. Moreover, each cluster possessed its own distinct functional enrichment terms. According to the algorithm prediction of the TIDE algorithm, the results show that the anti-PD1 immunotherapy response of Immunity_H patients is better than that of Immunity_L patients. Studies have also confirmed that Immunity_H can more express most HLA genes, which indicates that it is more immunogenic than other subtypes. Although the specific mechanism of immune escape is unknown, this finding provides a possible target for tailored patient therapy. The above discussion shows that the heterogeneity of the tumor immune microenvironment will produce different responses to immunotherapy or anti-cancer drugs. However, bioinformatics and further clinical studies are needed to confirm these results.

In conclusion, the identification of immune-associated $\mathrm{OvCa}$ subtypes has potential 


\section{clinical implications for OvCa therapy.}

\section{Reference:}

1. Bray F, Ferlay J, Soerjomataram I, et al. Global cancer statistics 2018: GLOBOCAN estimates of incidence and mortality worldwide for 36 cancers in 185 countries. CA Cancer J Clin 2018; 68: 394-424.

2. Siegel RL, Miller KD, Jemal A. Cancer statistics, 2019. CA Cancer J Clin 2019; 69: 7-34.

3. Jessmon P, Boulanger T, Zhou W, et al. Epidemiology and treatment patterns of epithelial ovarian cancer. Expert Rev Anticancer Ther 2017; 17: 427-437.

4. Perales-Puchalt A, Wojtak K, Duperret EK, et al. Engineered DNA Vaccination against Follicle-Stimulating Hormone Receptor Delays Ovarian Cancer Progression in Animal Models. Mol Ther2019; 27: 314-325.

5. Hoogstad-van Evert JS, Bekkers R, Ottevanger N, et al. Harnessing natural killer cells for the treatment of ovarian cancer. Gynecol Onco/2020; 157: 810-816.

6. Thomas ED, Meza-Perez S, Bevis KS, et al. IL-12 Expressing oncolytic herpes simplex virus promotes anti-tumor activity and immunologic control of metastatic ovarian cancer in mice. J Ovarian Res 2016; 9: 70.

7. Maby P, Corneau A, Galon J. Phenotyping of tumor infiltrating immune cells using mass cytometry (CyTOF). Methods Enzymo/2020; 632: 339-368.

8. Kather JN, Horner C, Weis CA, et al. CD163+ immune cell infiltrates and presence of CD54+ microvessels are prognostic markers for patients with embryonal rhabdomyosarcoma. Sci Rep 2019; 9: 9211.

9. Chifman J, Pullikuth A, Chou JW, et al. Conservation of immune gene signatures in solid tumors and prognostic implications. BMC Cancer2016; 16: 911.

10. Tu L, Guan R, Yang $\mathrm{H}$, et al. Assessment of the expression of the immune checkpoint molecules PD-1, CTLA4, TIM-3 and LAG-3 across different cancers in relation to treatment response, tumor-infiltrating immune cells and survival. Int J Cancer 2020; 147: 423-439.

11. MacGregor HL, Sayad A, Elia A, et al. High expression of B7-H3 on stromal cells defines tumor and stromal compartments in epithelial ovarian cancer and is associated with limited immune activation. J Immunother Cancer 2019; 7: 357.

12. Kwiatkowska D, Kluska P, Reich A. Beyond PD-1 Immunotherapy in Malignant Melanoma. 
Dermatologyand therapy 2019; 9: 243-257.

13. Koller KM, Wang W, Schell TD, et al. Malignant melanoma-The cradle of anti-neoplastic immunotherapy. Critical reviews in oncology/hematology 2016; 106: 25-54.

14. Odunsi K. Immunotherapy in ovarian cancer. Ann Onco/2017; 28: viii1-viii7.

15. Wang W, Liu JR, Zou W. Immunotherapy in Ovarian Cancer. Surg Oncol Clin N Am 2019; 28: $447-464$.

16. Hanzelmann S, Castelo R, Guinney J. GSVA: gene set variation analysis for microarray and RNA-seq data. BMC Bioinformatics 2013; $14: 7$.

17. Krug K, Mertins P, Zhang B, et al. A Curated Resource for Phosphosite-specific Signature Analysis. Mol Cel/ Proteomics 2019; 18: 576-593.

18. Yoshihara K, Shahmoradgoli M, Martinez E, et al. Inferring tumour purity and stromal and immune cell admixture from expression data. Nat Commun2013; 4: 2612.

19. Newman AM, Liu CL, Green MR, et al. Robust enumeration of cell subsets from tissue expression profiles. Nature methods 2015; 12: 453-457.

20. Subramanian A, Tamayo P, Mootha VK, et al. Gene set enrichment analysis: a knowledgebased approach for interpreting genome-wide expression profiles. Proc Natl Acad Sci U SA 2005; 102: 15545-15550.

21. Mayakonda A, Koeffler HP. Maftools: Efficient analysis, visualization and summarization of MAF files from large-scale cohort based cancer studies. BioRxiv2016: 052662.

22. Jiang P, Gu S, Pan D, et al. Signatures of T cell dysfunction and exclusion predict cancer immunotherapy response. Nature medicine 2018; 24: 1550-1558.

23. Hoshida Y, Brunet JP, Tamayo P, et al. Subclass mapping: identifying common subtypes in independent disease data sets. PloS one 2007; 2: e1195.

24. Yang W, Soares J, Greninger P, et al. Genomics of Drug Sensitivity in Cancer (GDSC): a resource for therapeutic biomarker discovery in cancer cells. Nucleic acids research2013; 41: D955-961.

25. Hazra A, Gogtay N. Biostatistics Series Module 3: Comparing Groups: Numerical Variables. Indian journal of dermatology 2016; 61: 251-260.

26. Bland JM, Altman DG. Survival probabilities (the Kaplan-Meier method). Bmj 1998; 317: 1572. 
27. Althaf MM, El Kossi M, Jin JK, et al. Human leukocyte antigen typing and crossmatch: A comprehensive review. World J Transplant2017; 7: 339-348.

28. van Drongelen V, Holoshitz J. Human Leukocyte Antigen-Disease Associations in Rheumatoid Arthritis. Rheum Dis Clin North Am 2017; 43: 363-376.

29. Bindea G, Mlecnik B, Tosolini M, et al. Spatiotemporal dynamics of intratumoral immune cells reveal the immune landscape in human cancer. Immunity 2013; 39: 782-795.

30. Patel SP, Kurzrock R. PD-L1 Expression as a Predictive Biomarker in Cancer Immunotherapy. Mol Cancer Ther 2015; 14: 847-856.

31. Weidle UH, Birzele F, Kollmorgen G, et al. Mechanisms and Targets Involved in Dissemination of Ovarian Cancer. Cancer Genomics Proteomics 2016; 13: 407-423.

32. De La Motte Rouge T, Corne J, Cauchois A, et al. Serum CD95L Level Correlates with Tumor Immune Infiltration and Is a Positive Prognostic Marker for Advanced High-Grade Serous Ovarian Cancer. Mol Cancer Res 2019; 17: 2537-2548.

33. Liu J, Tan Z, He J, et al. Identification of three molecular subtypes based on immune infiltration in ovarian cancer and its prognostic value. Biosci Rep 2020.

34. Alcaraz-Sanabria A, Baliu-Pique M, Saiz-Ladera C, et al. Genomic Signatures of Immune Activation Predict Outcome in Advanced Stages of Ovarian Cancer and Basal-Like Breast Tumors. Front Onco/2019; 9: 1486.

35. Wang J, Chen $\mathrm{H}$, Chen X, et al. Expression of Tumor-Related Macrophages and Cytokines After Surgery of Triple-Negative Breast Cancer Patients and its Implications. Med SCi Monit 2016; 22: 115-120.

36. Mantovani A, Sozzani S, Locati M, et al. Macrophage polarization: tumor-associated macrophages as a paradigm for polarized M2 mononuclear phagocytes. Trends Immunol 2002; 23: 549-555.

37. Guo L, Zhang Y, Yang Z, et al. Tunneling Nanotubular Expressways for Ultrafast and Accurate M1 Macrophage Delivery of Anticancer Drugs to Metastatic Ovarian Carcinoma. ACS Nano 2019; 13: 1078-1096.

38. An Y, Yang Q. MiR-21 modulates the polarization of macrophages and increases the effects of M2 macrophages on promoting the chemoresistance of ovarian cancer. Life SCi 2020; 242: 117162.

39. Novy P, Quigley M, Huang X, et al. CD4 T cells are required for CD8 T cell survival during 
both primary and memory recall responses. J Immuno/2007; 179: 8243-8251.

40. Li J, Li X, Zhang C, et al. A signature of tumor immune microenvironment genes associated with the prognosis of nonsmall cell lung cancer. Oncol Rep 2020; 43: 795-806.

41. Palmirotta R, Silvestris E, D'Oronzo S, et al. Ovarian cancer: Novel molecular aspects for clinical assessment. CritRev Oncol Hemato/2017; 117: 12-29.

42. Talhouk A, George J, Wang C, et al. Development and Validation of the Gene Expression Predictor of High-grade Serous Ovarian Carcinoma Molecular SubTYPE (PrOTYPE). Clin Cancer Res 2020; 26: 5411-5423.

43. Cancer Genome Atlas Research N. Integrated genomic analyses of ovarian carcinoma. Nature 2011; 474: 609-615.

\section{Declarations section}

\section{Ethics approval and consent to participate}

Not applicable.

\section{Consent for publication}

Not applicable.

\section{Availability of data and material}

Data availability could be obtained from the TCGA website.

\section{Competing interests}

The authors declare that they have no competing interests.

\section{Funding}

None.

\section{Author contribution statement:}

L.Y; J.K.S; Q.A wrote the main manuscript text;

T.L; L.Y; J.K.S prepared Figures 1-10;

J.K.S; T.L contributed to data analysis; 
All authors reviewed the manuscript.

\section{Acknowledgment}

None.

Figure legends:
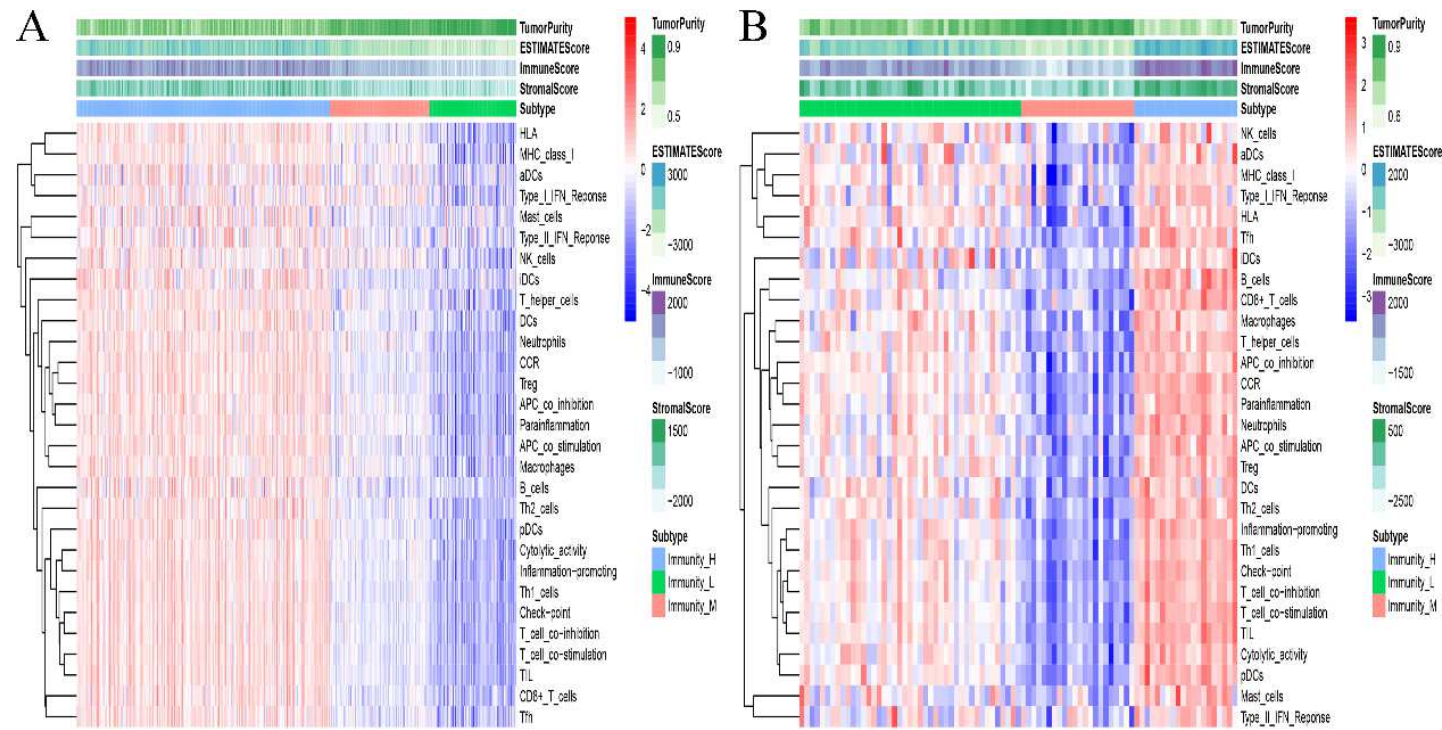

Figure 1. Three clusters were identified by the unsupervised hierarchical clustering in two publicly datasets. A, B: TCGA and ICGC OvCa cohort. 

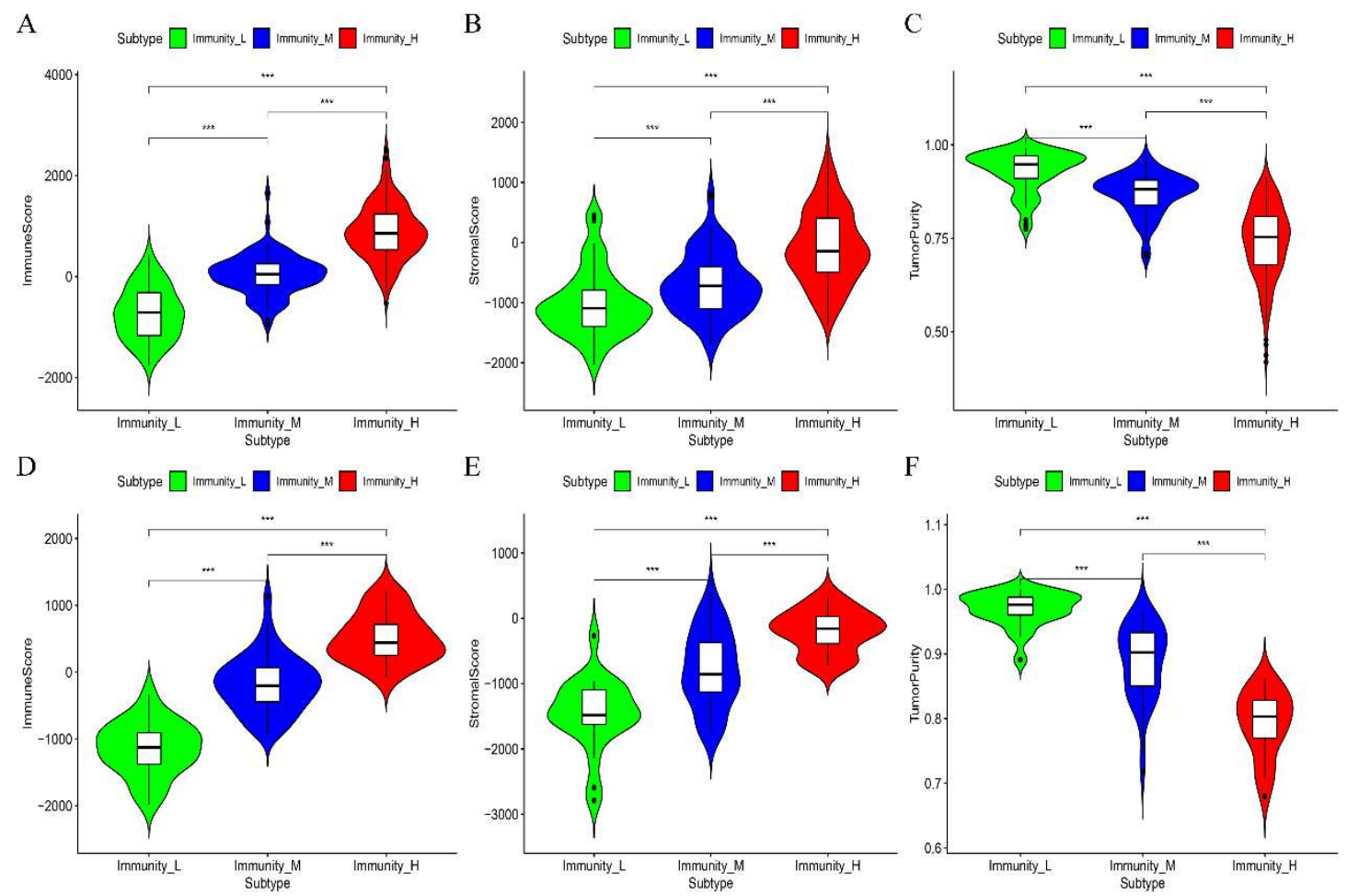

Figure 2. The Immune Score, Stromal Score, and Tumor Purity were compared among three OvCa subgroups. A-F: Immune Score, Stromal Score, and Tumor Purity in the TCGA and ICGC OvCa cohort, respectively.

A

Survival curve $(p=0.036)$

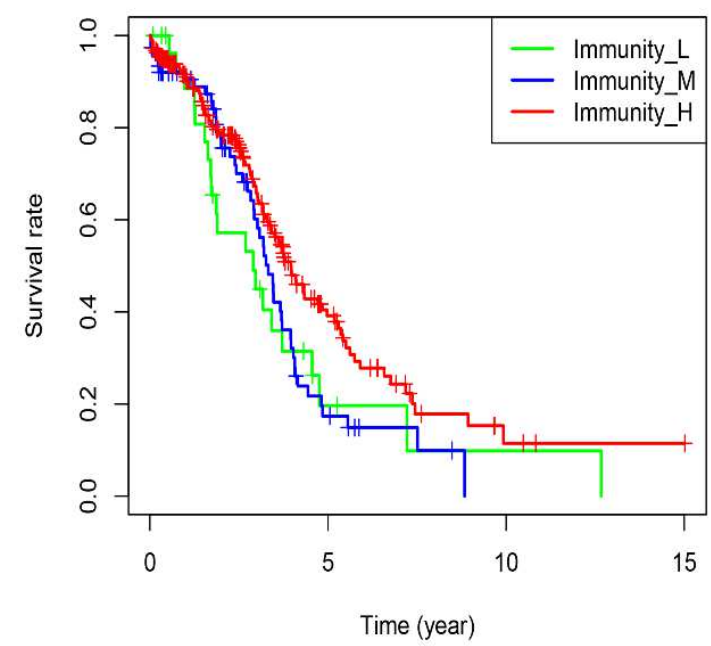

B

Survival curve $(p=0.065)$

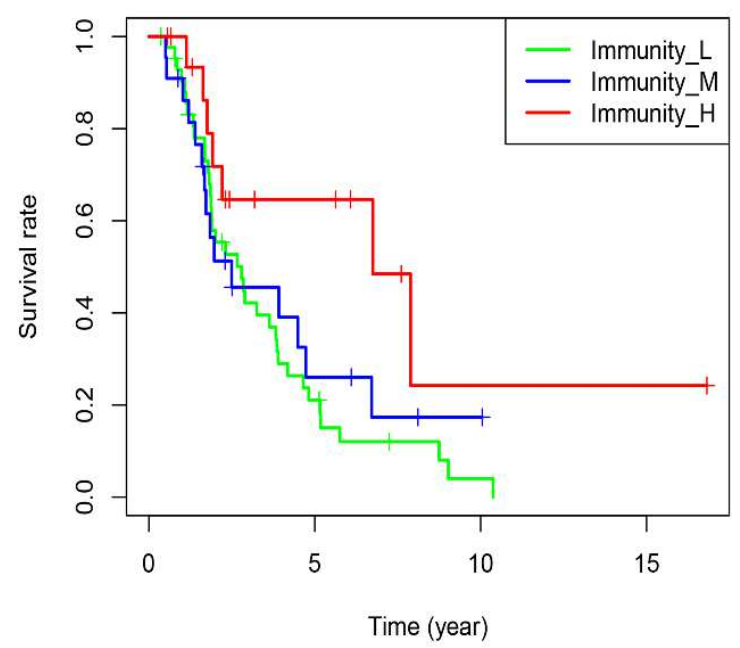

Figure 3. The expression of HLA related genes (A, C: TCGA and ICGC OvCa cohort) and immune cell subpopulation marker genes (B, D: TCGA and ICGC OvCa cohort) were compared among three PCa subtypes. 
A

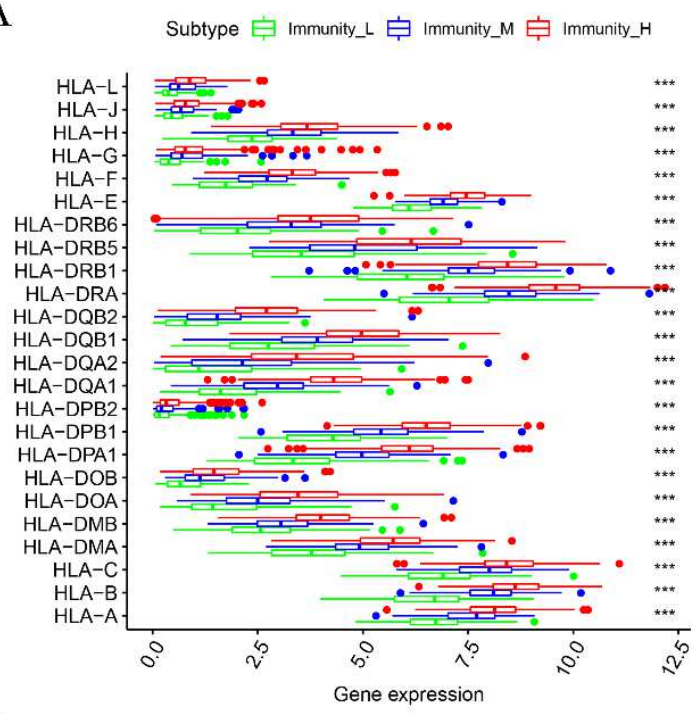

$\mathrm{C}$

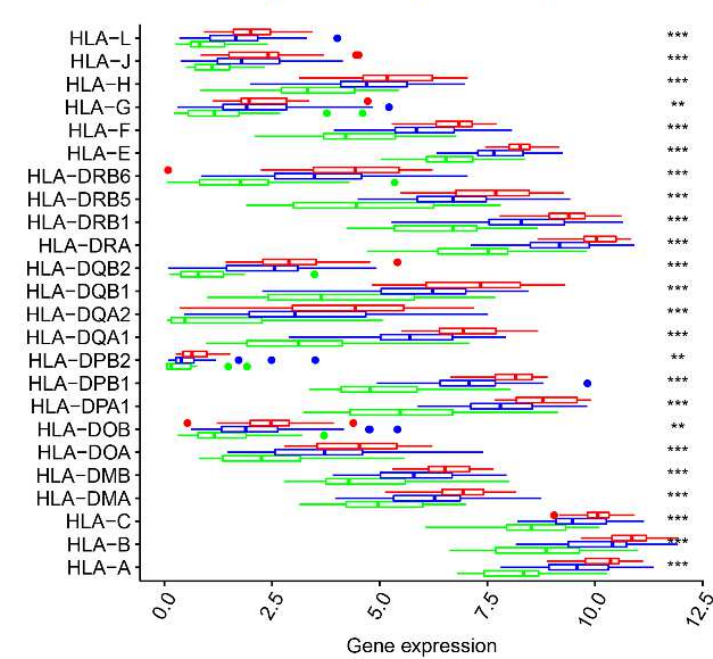

B

Subtype 户 Immunity_L 户 Immunity_M 官 Immunity_H

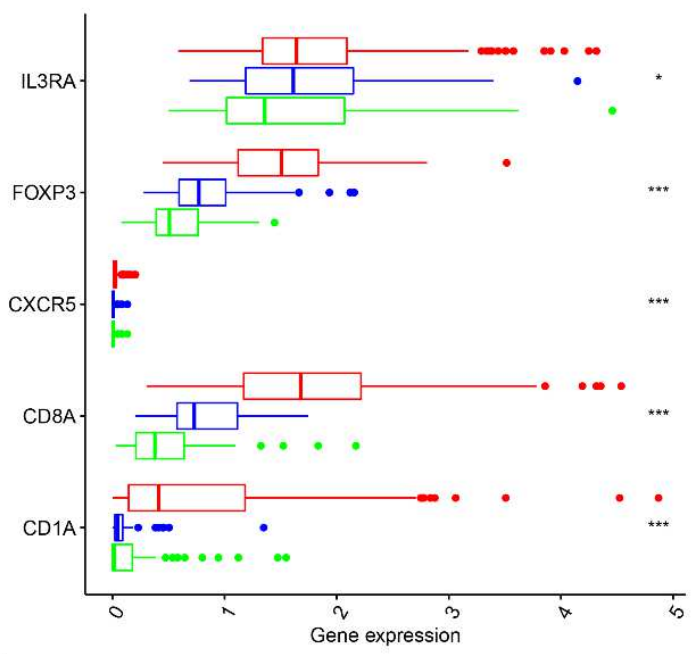

$\mathrm{D}$

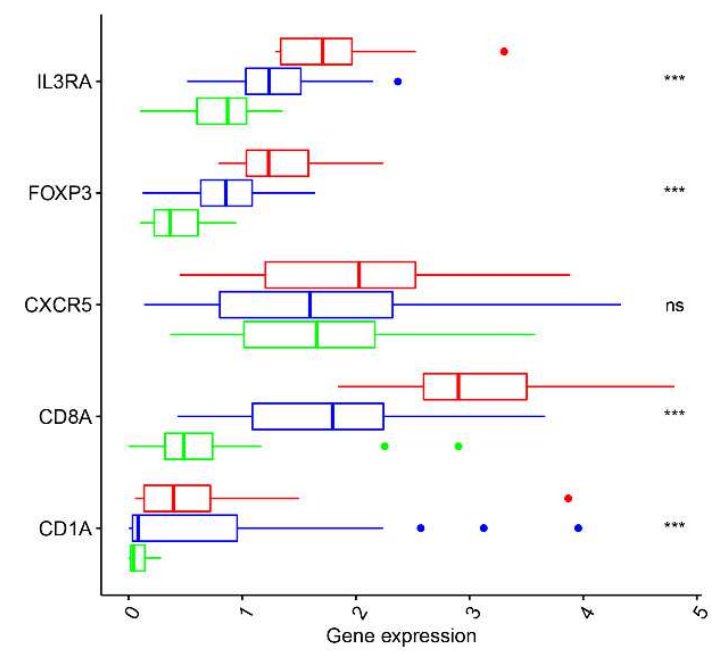

Figure 4. The expression of PD-1, PD-L1, and PD-L2 was compared among three OvCa subtypes in TCGA (A, B, C) and ICGC (D, E, F) datasets. 

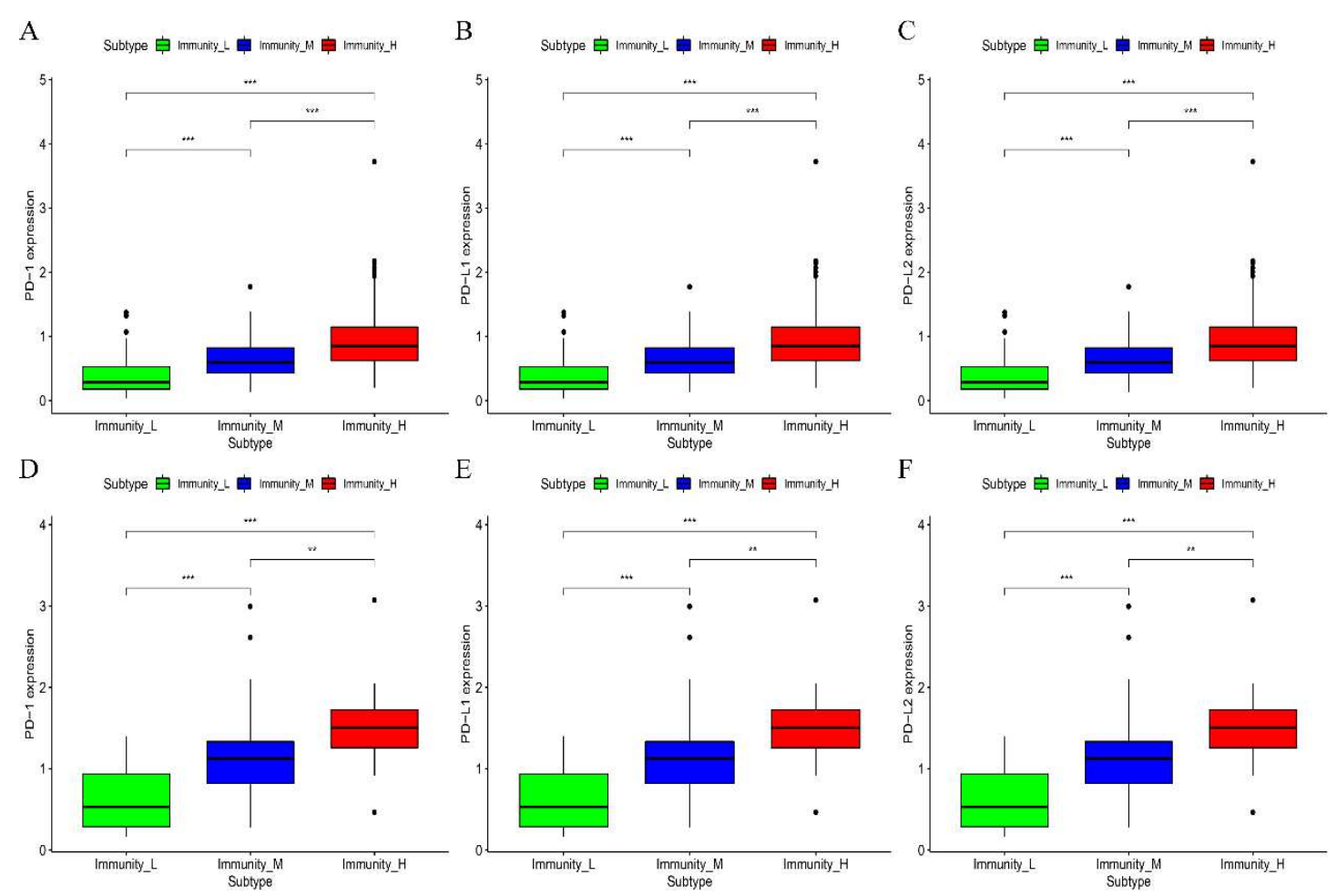

Figure 5. Kaplan-Meier curves were plotted to exhibit differences in the TCGA (A) and ICGC (B) datasets.

A

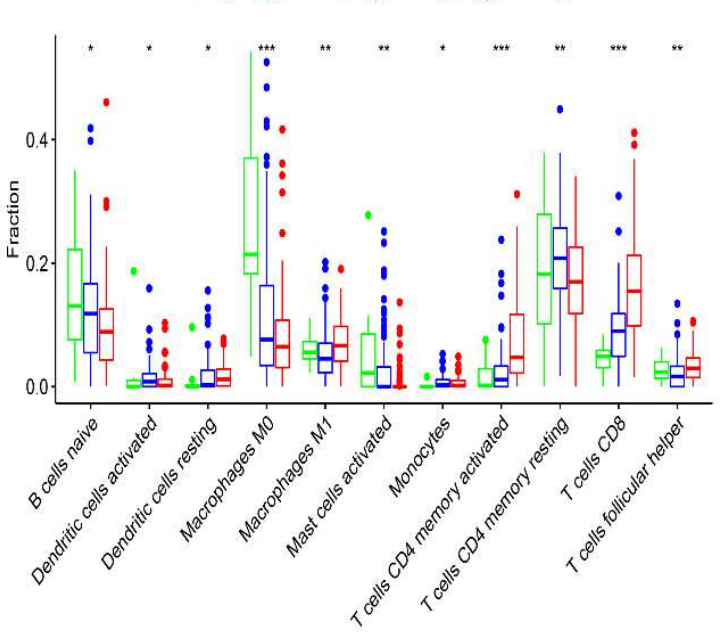

B

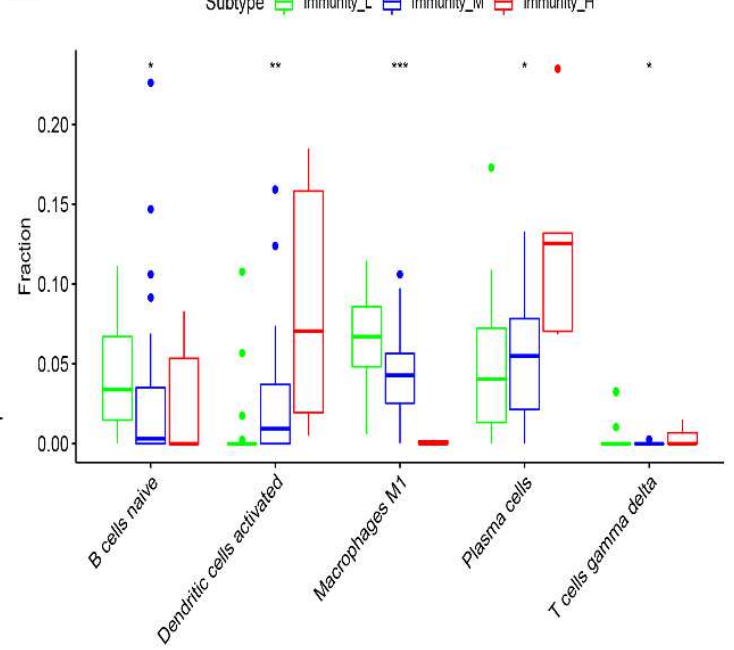

Figure 6. Compared the differences in the composition of immune cell subgroups between the three OvCa subtypes in the TCGA (A) and ICGC (B) data sets. 

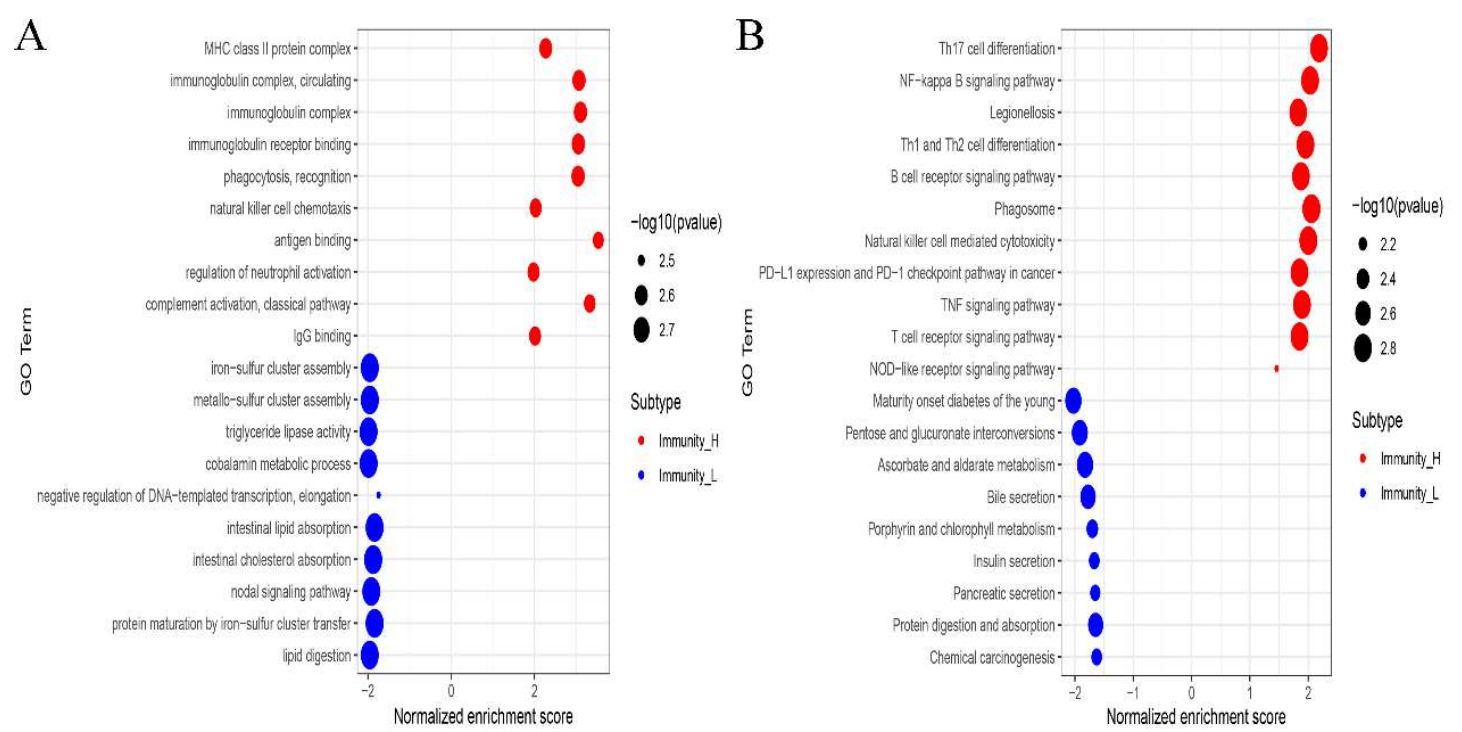

Figure 7. Identification of OvCa subtype-specific up-regulation of GO (A) and KEGG terms (B) among three OvCa subtypes using the GSEA method in the TCGA dataset. (C). GSVA exhibits differences in the KEGG pathway between the Immunity_H and Immunity_Lsubgroups of the TCGA dataset.

A

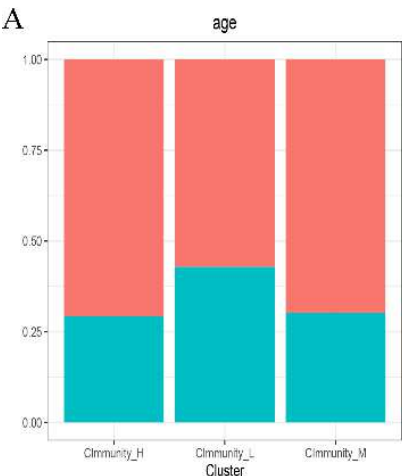

$\mathrm{D}$

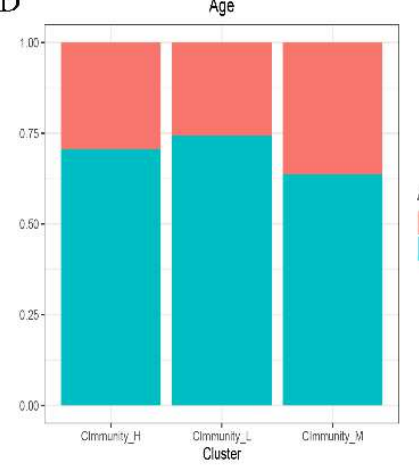

B

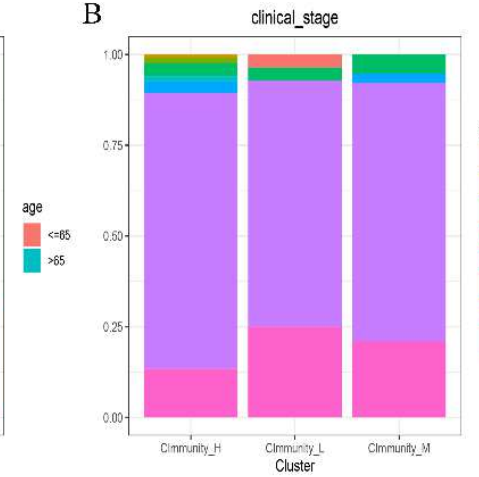

E

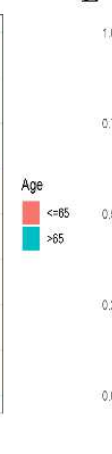<smiles>[CH]1CCC1</smiles>

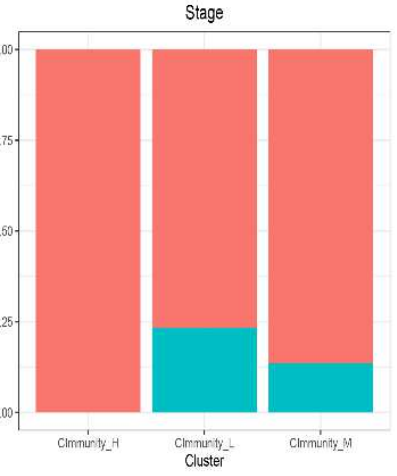

C
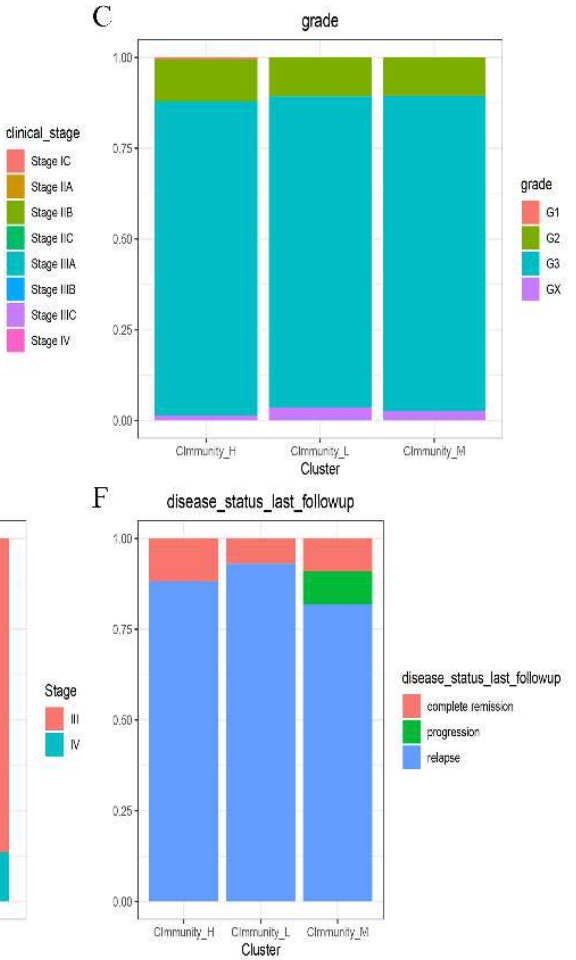

Figure 8. Comparison of clinical features between the Immune-H and Immune-L OvCa subsets in the TCGA and ICGC datasets. Age (A), FIGO TNM stage (B), Grade (C) distributions for each subtype in the TCGA dataset, Age (D), FIGO TNM stage (E), disease status last followup (F) in the ICGC dataset. 


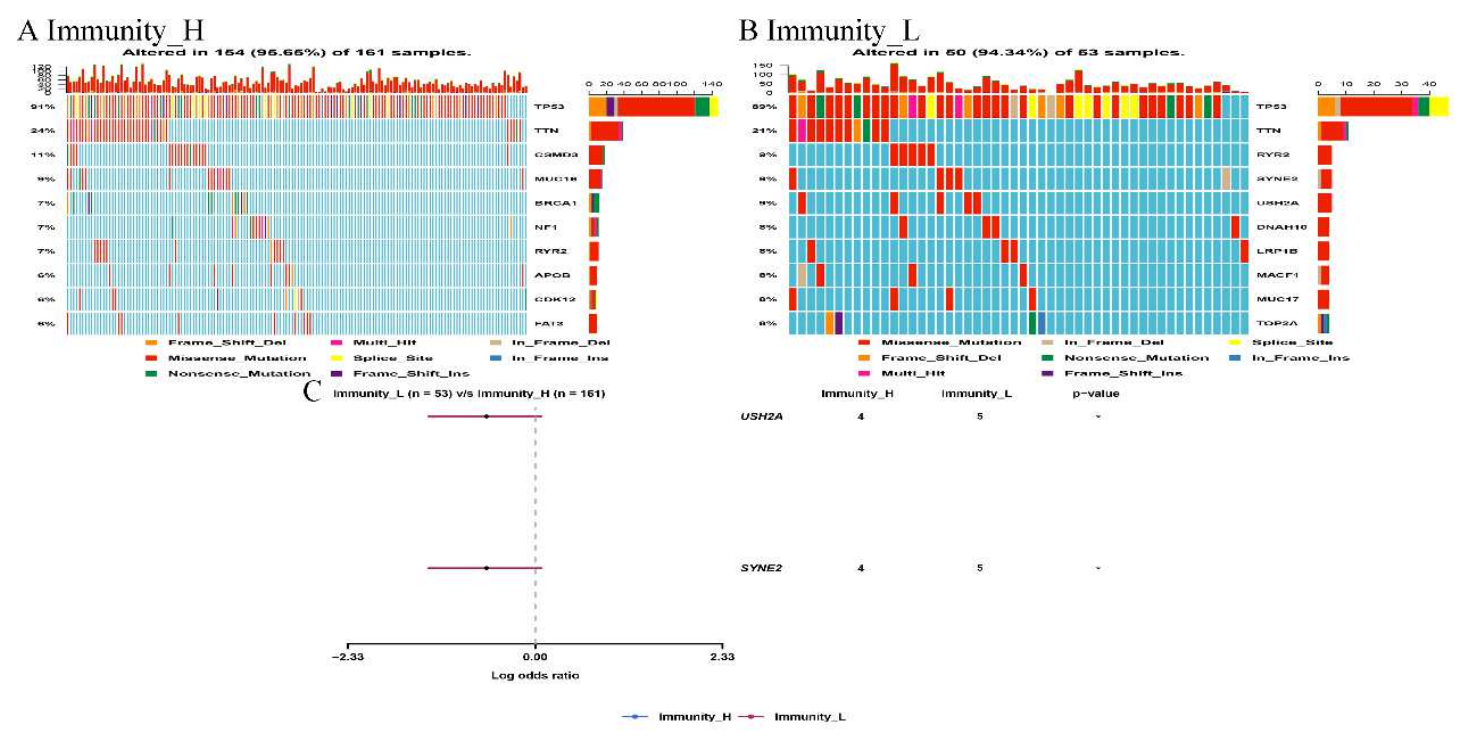

Figure 9. (A) Comparison of OvCa subtype-specific gene mutations between the Immunity_H and Immunity_Lsubsets in the TCGA dataset. (B) Gene mutation data of highly mutated genes between the Immune-H and Immune-L OvCa subsets. (C) The forest plot exhibits the comparison results of gene mutations $(* \mathrm{P}, 0.1, * * \mathrm{P}, 0.05$, ns: not significant).

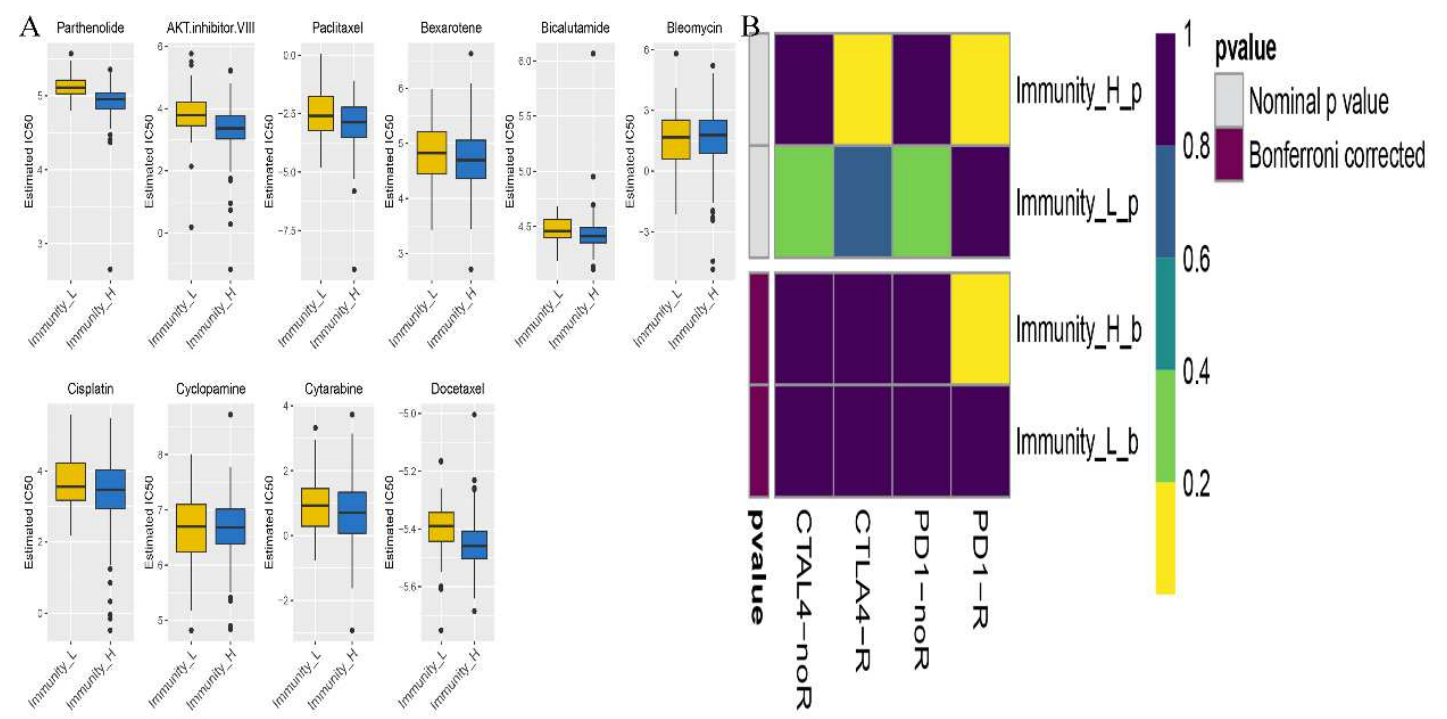

Figure 10. Prediction for Immunotherapy and Anti-cancer Drug Response. The box plots show the estimated $\mathrm{IC}_{50}$ for chemotherapeutic drugs using the GDSC database: (A) for Immune-H and Immune-L OvCa subsets, (B) Submap algorithm demonstrated that Immune-H is more sensitive to the immunotherapy (Bonferroni-corrected $\mathrm{P}<0.05$ ). 
Figures
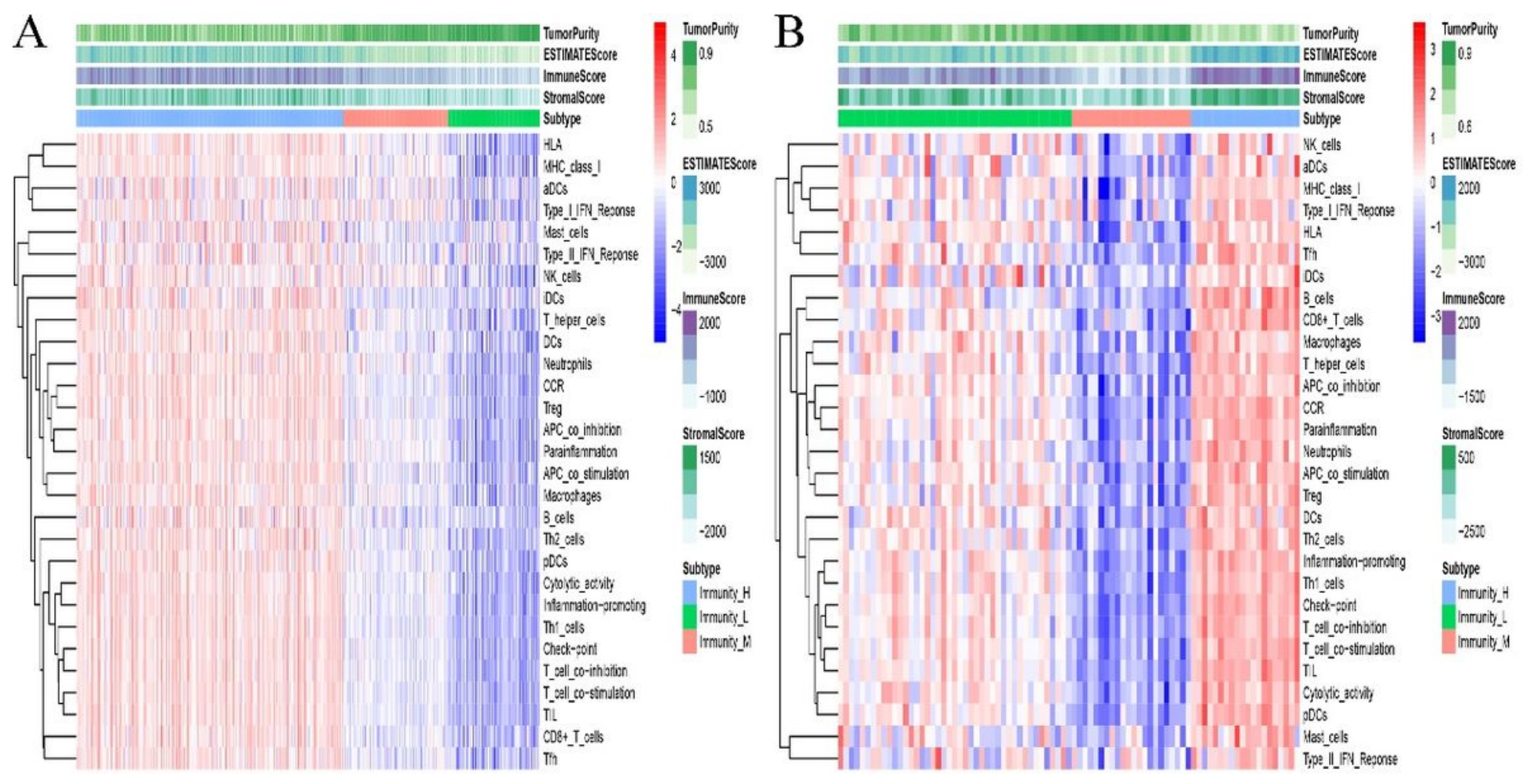

Figure 1

Three clusters were identified by the unsupervised hierarchical clustering in two publicly datasets. A, B: TCGA and ICGC OvCa cohort. 


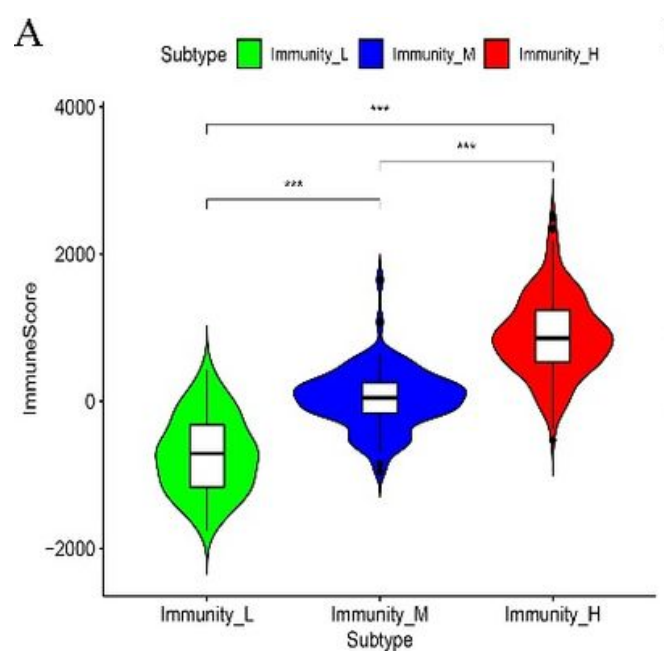

D

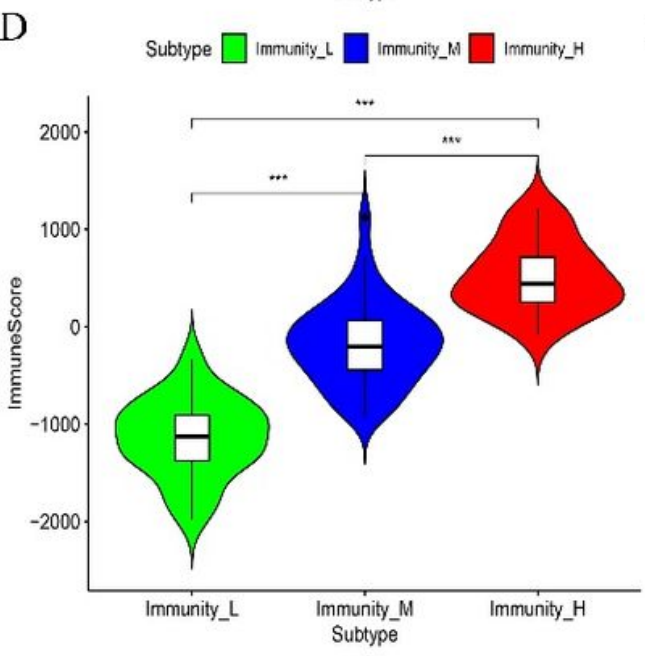

B

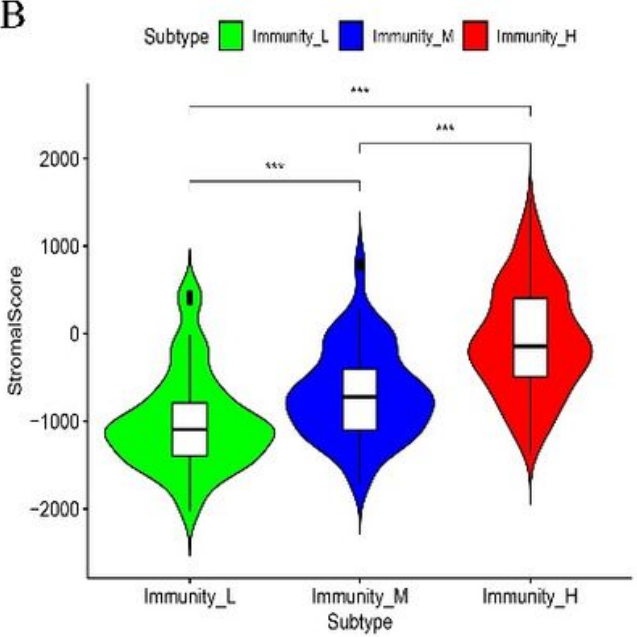

$\mathrm{E}$

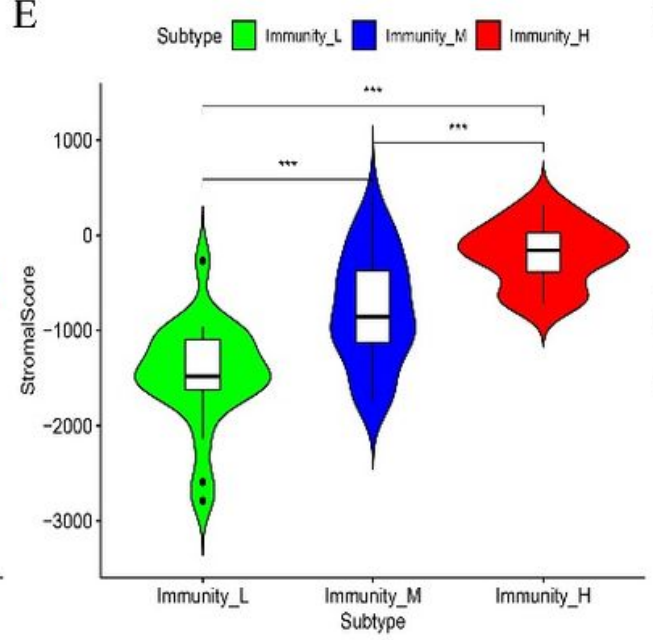

C Subype $\square$ Immunty_L $\square$ Immunity_M $\square$ Immunity_H

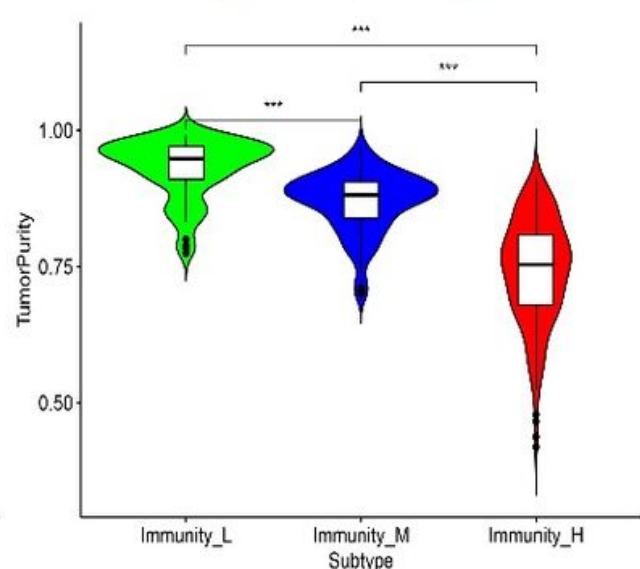

F

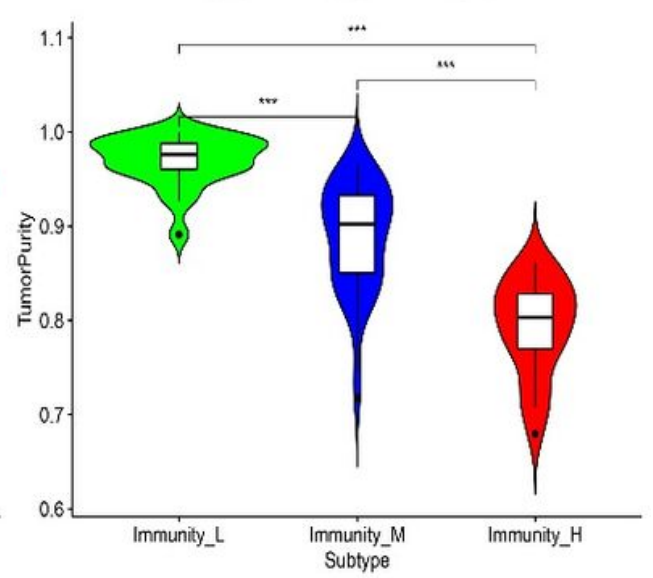

Figure 2

The Immune Score, Stromal Score, and Tumor Purity were compared among three OvCa subgroups. A-F: Immune Score, Stromal Score, and Tumor Purity in the TCGA and ICGC OvCa cohort, respectively. 
A

Survival curve $(p=0.036)$

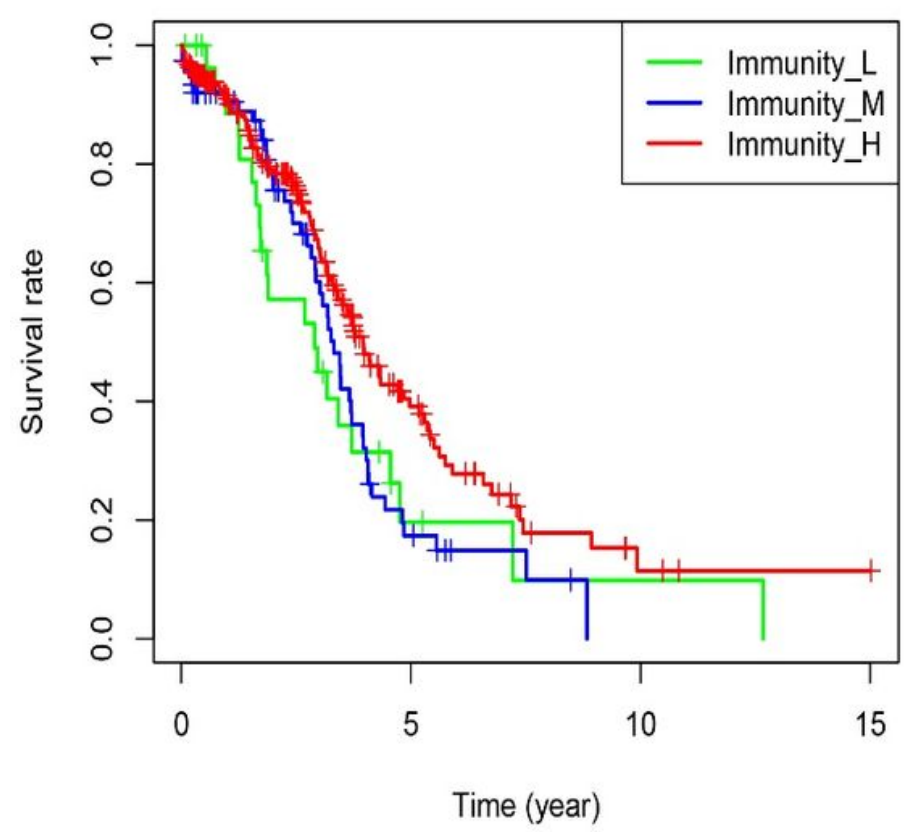

B

Survival curve $(p=0.065)$

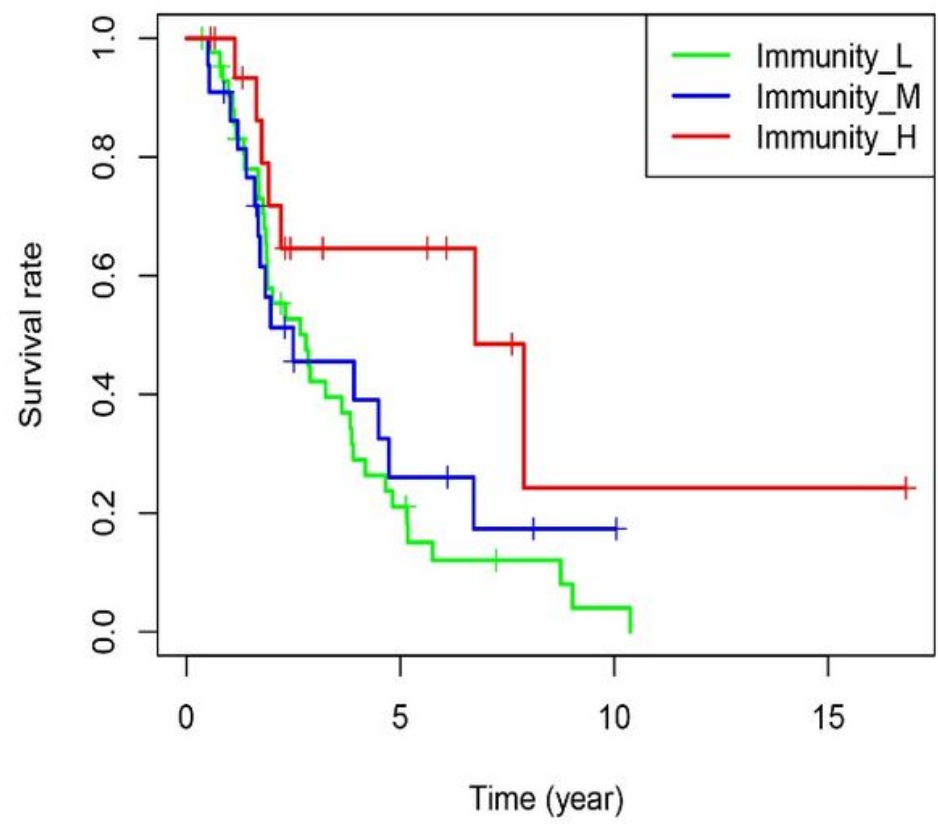

\section{Figure 3}

The expression of HLA related genes (A, C: TCGA and ICGC OvCa cohort) and immune cell subpopulation marker genes (B, D: TCGA and ICGC OvCa cohort) were compared among three PCa subtypes. 
A

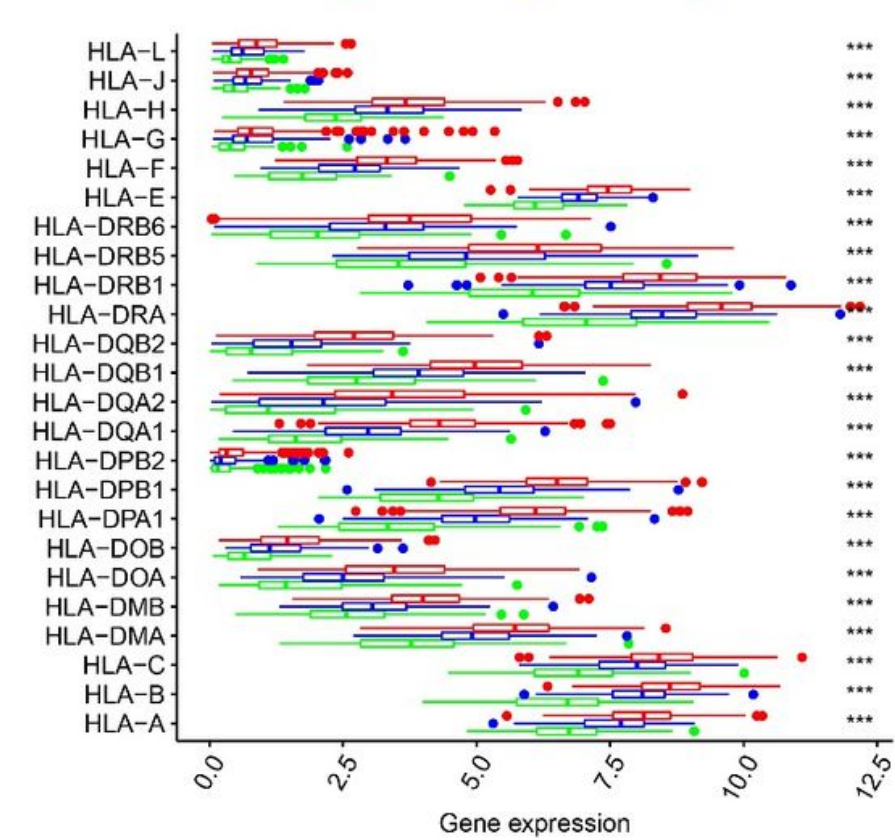

$\mathrm{C}$

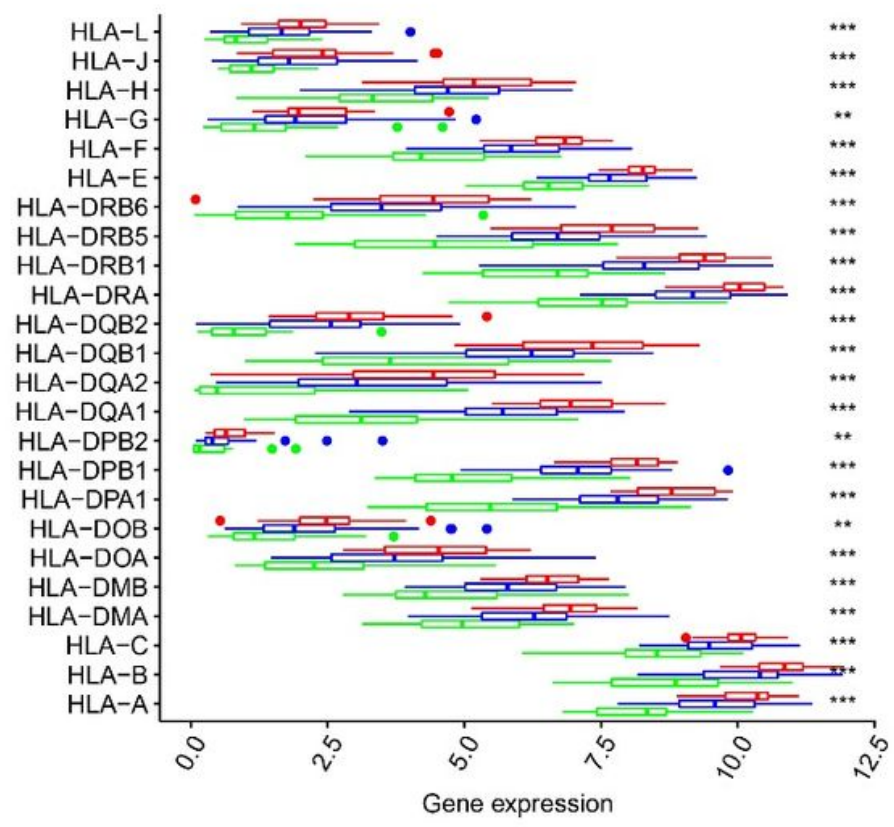

B

Subtype 官 Immunity_L 官 Immunity_M 官 Immunity_H

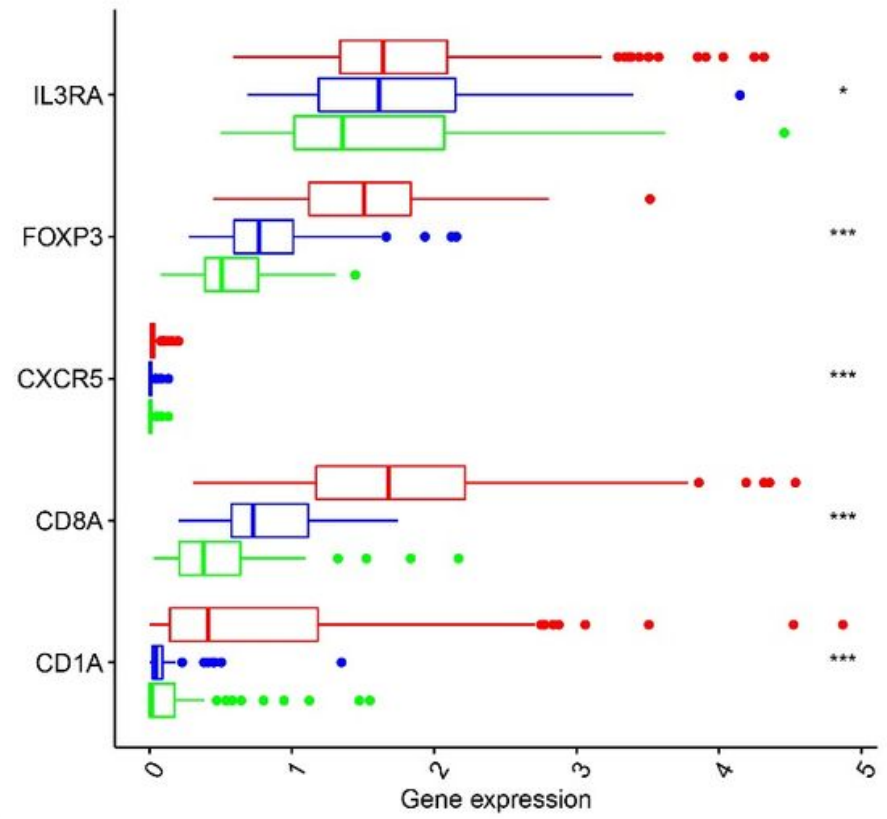

$\mathrm{D}$

Subtype 官 Immunity_L 追 Immunity_M 官 Immunity_H

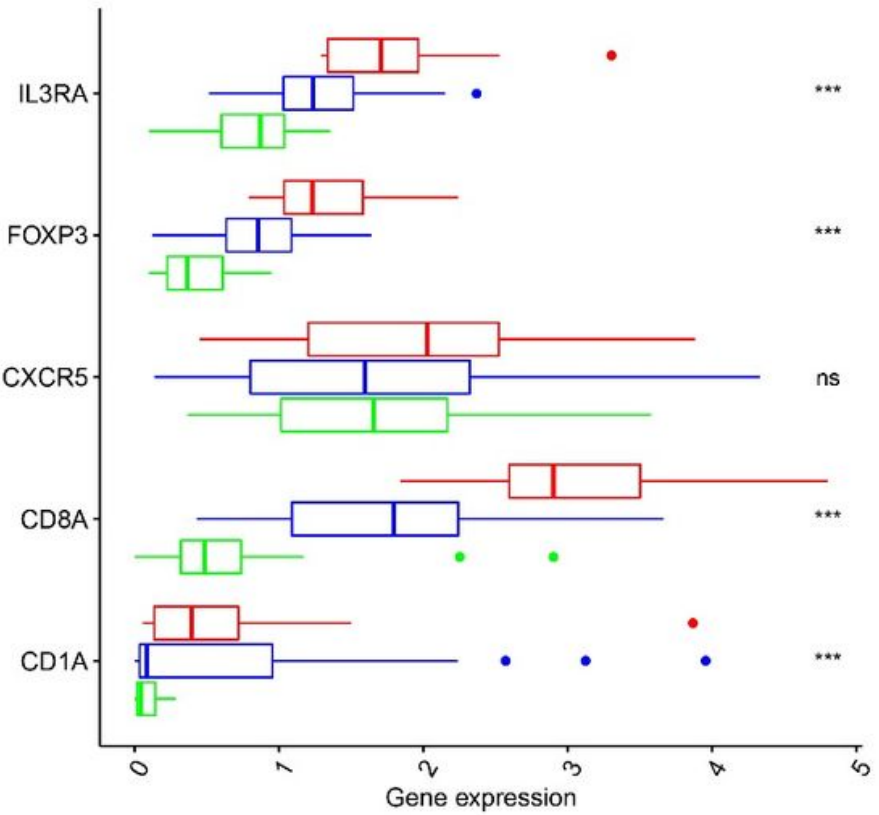

Figure 4

The expression of PD-1, PD-L1, and PD-L2 was compared among three OvCa subtypes in TCGA (A, B, C) and ICGC (D, E, F) datasets. 

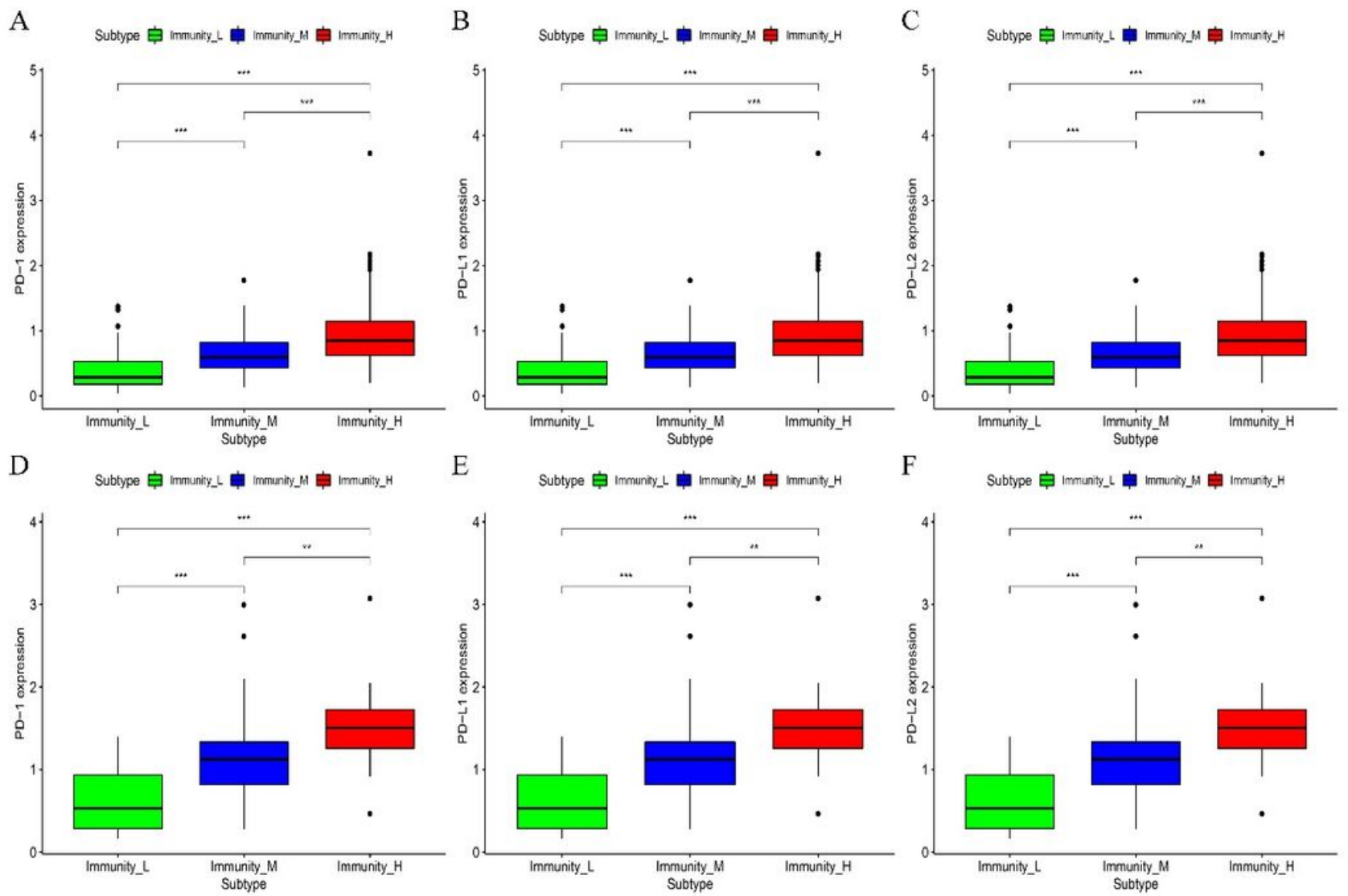

\section{Figure 5}

Kaplan-Meier curves were plotted to exhibit differences in the TCGA (A) and ICGC (B) datasets. 

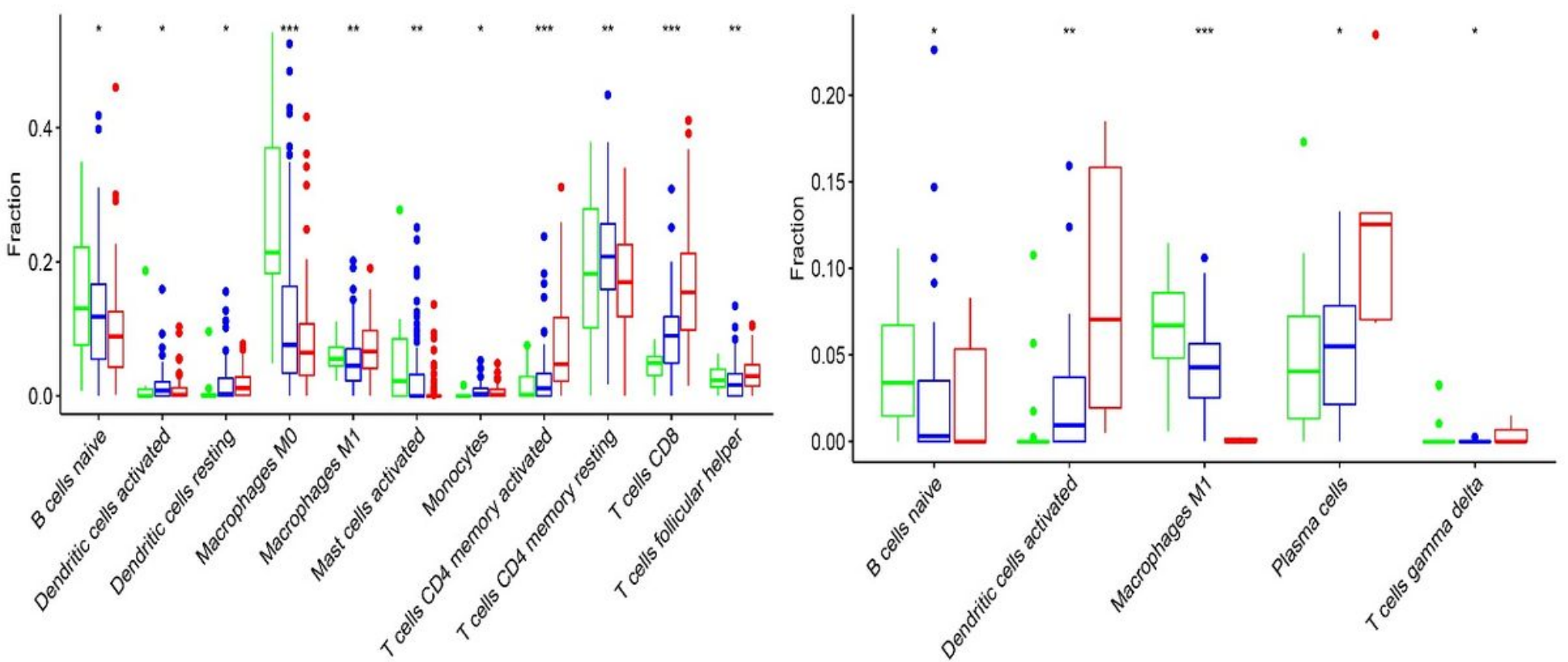

Figure 6

Compared the differences in the composition of immune cell subgroups between the three OvCa subtypes in the TCGA (A) and ICGC (B) data sets.

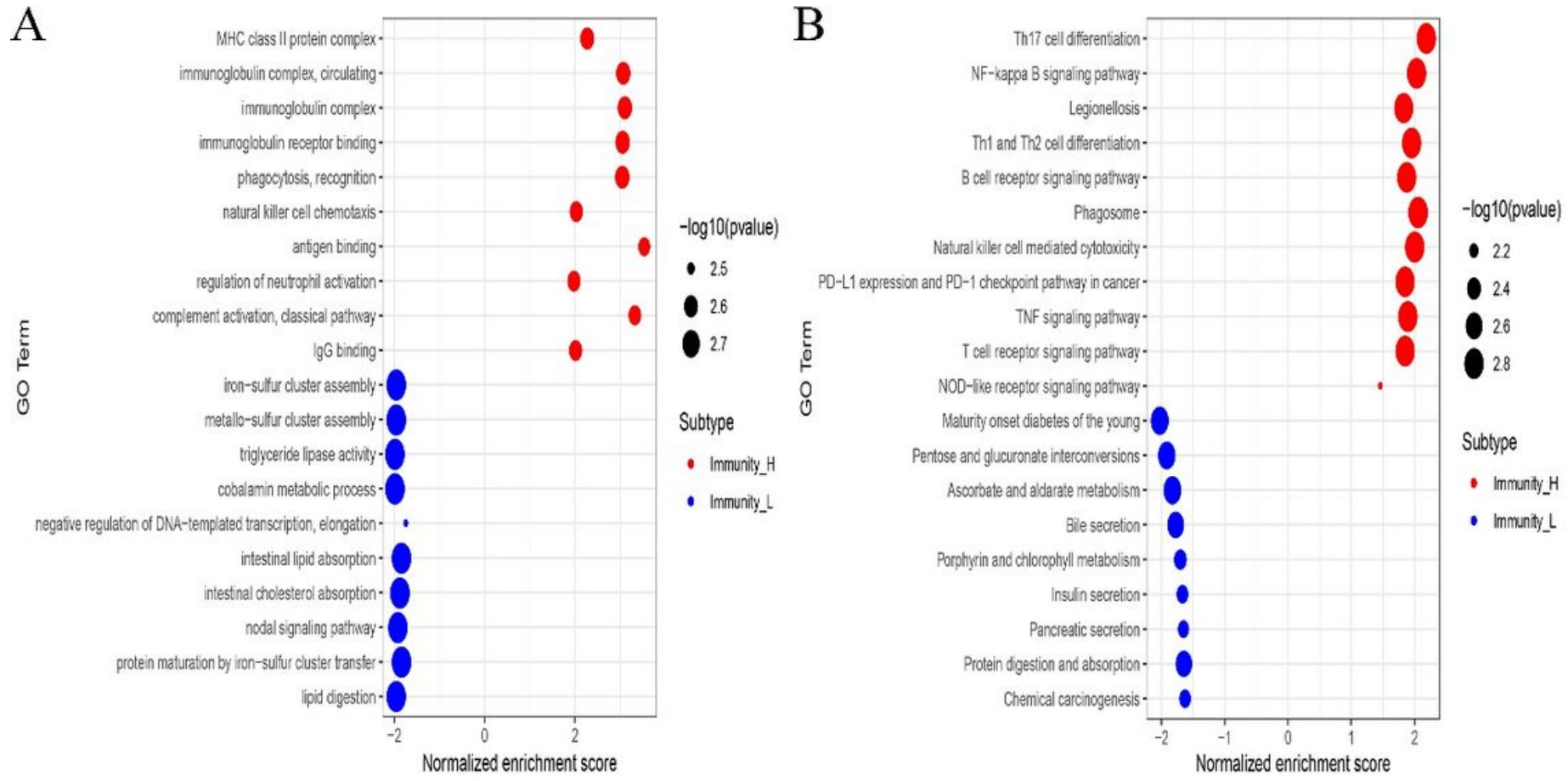

Figure 7 
Identification of OvCa subtype-specific up-regulation of GO (A) and KEGG terms (B) among three OvCa subtypes using the GSEA method in the TCGA dataset. (C). GSVA exhibits differences in the KEGG pathway between the Immunity_H and Immunity_L subgroups of the TCGA dataset.

A

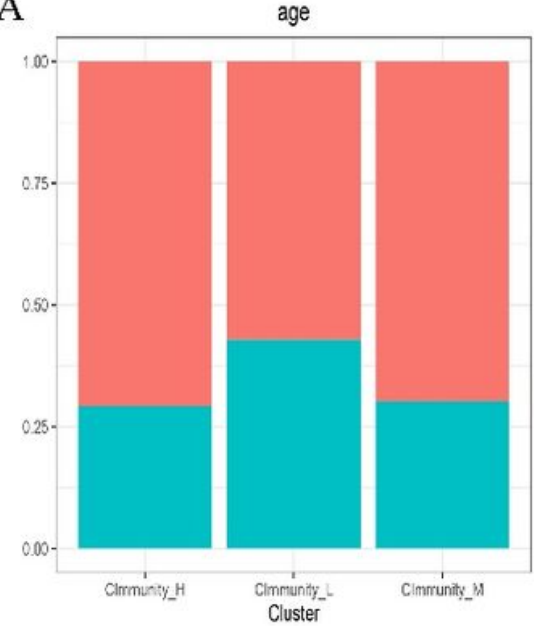

$\mathrm{D}$

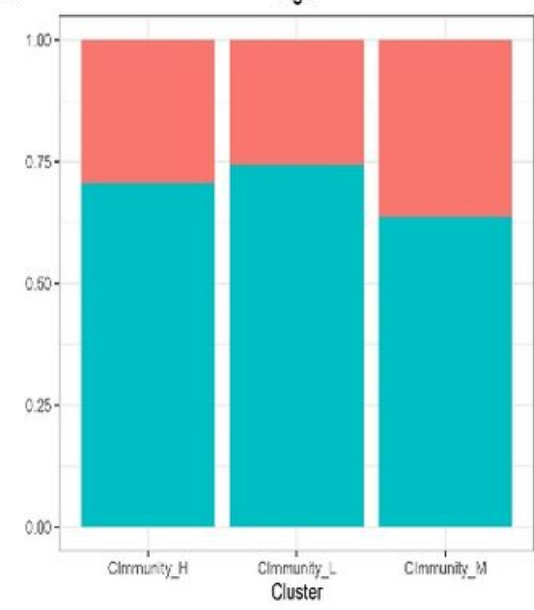

B

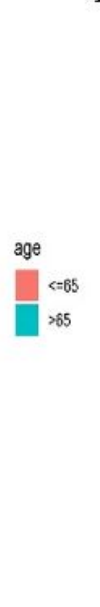

E

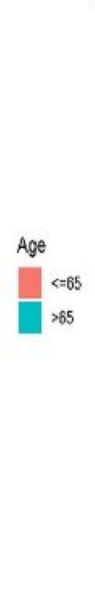

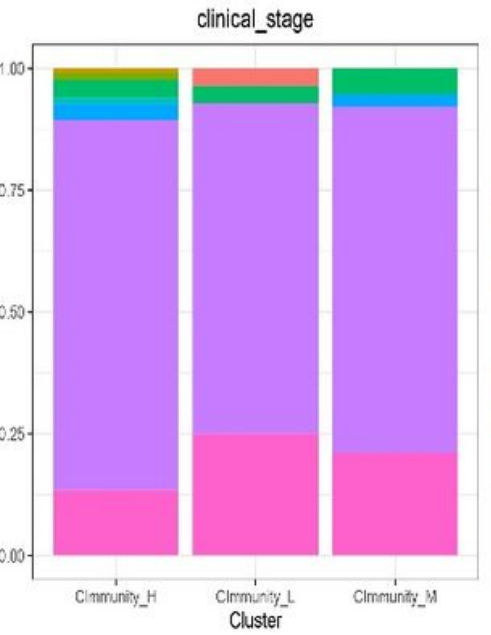

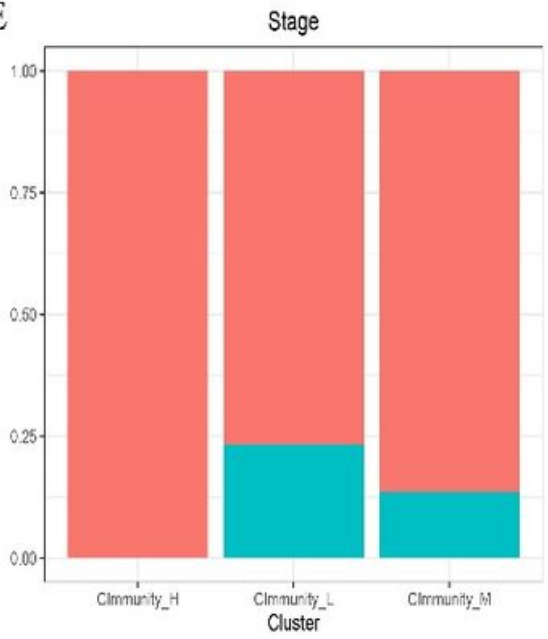

C
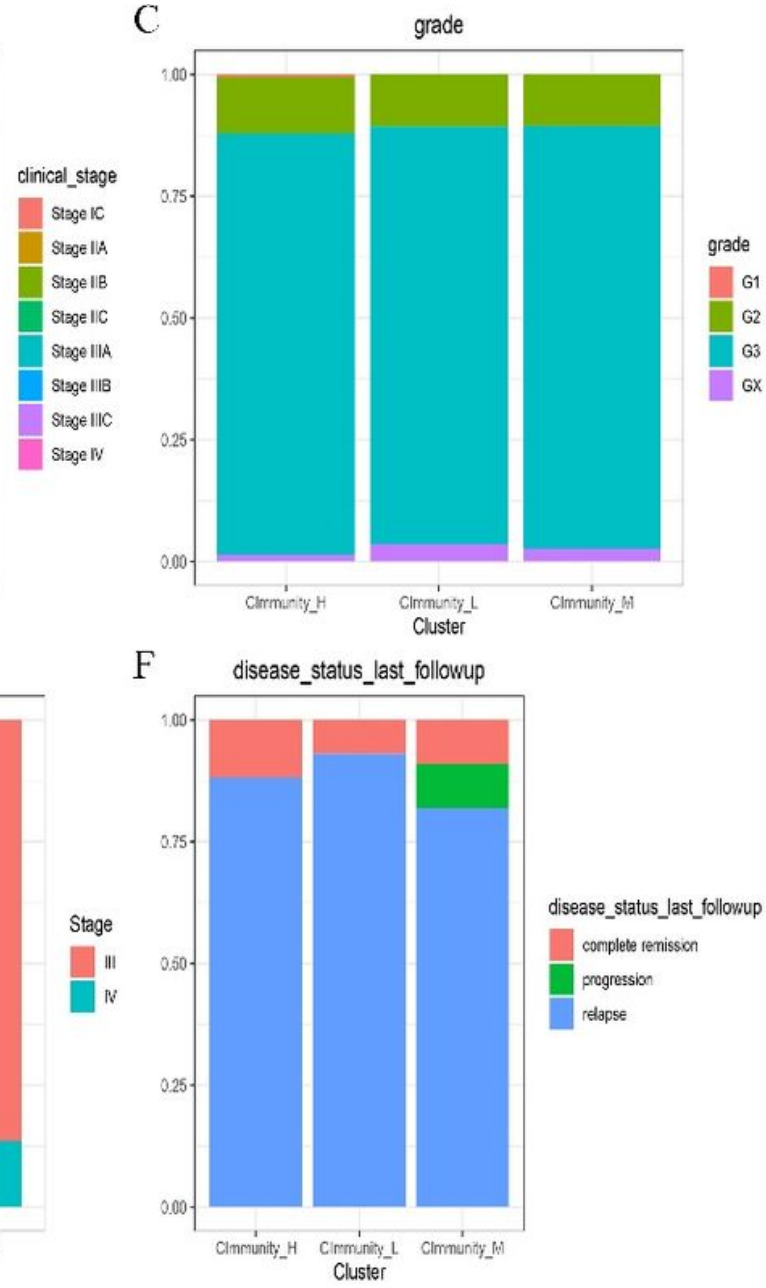

\section{Figure 8}

Comparison of clinical features between the Immune-H and Immune-L OvCa subsets in the TCGA and ICGC datasets. Age (A), FIGO TNM stage (B), Grade (C) distributions for each subtype in the TCGA dataset, Age (D), FIGO TNM stage (E), disease status last followup (F) in the ICGC dataset. 
A Immunity_H

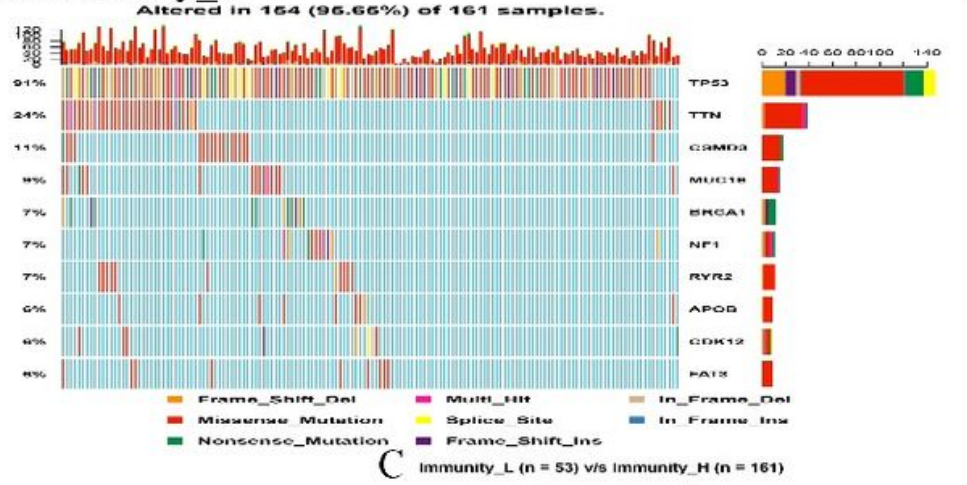

Cimmunity_L $(n=53)$ v/s immunity_H ( $n=161)$

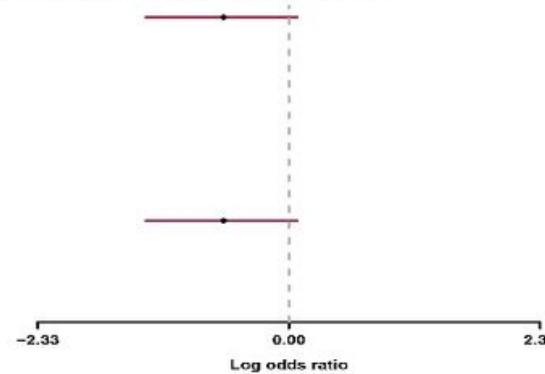

B Immunity_L

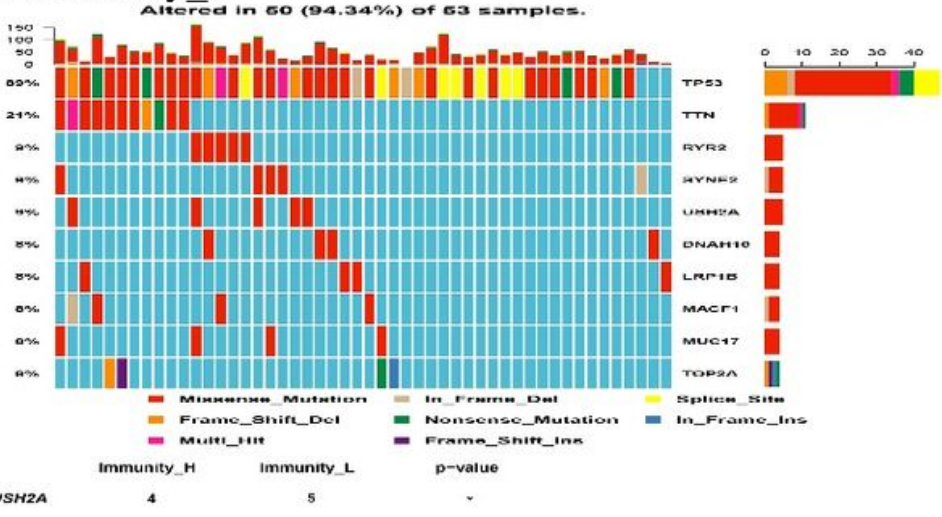

\section{Figure 9}

(A) Comparison of OvCa subtype-specific gene mutations between the Immunity_H and Immunity_L subsets in the TCGA dataset. (B) Gene mutation data of highly mutated genes between the Immune-H and Immune-L OvCa subsets. (C) The forest plot exhibits the comparison results of gene mutations (*P, $0.1, * * P, 0.05$, ns: not significant).
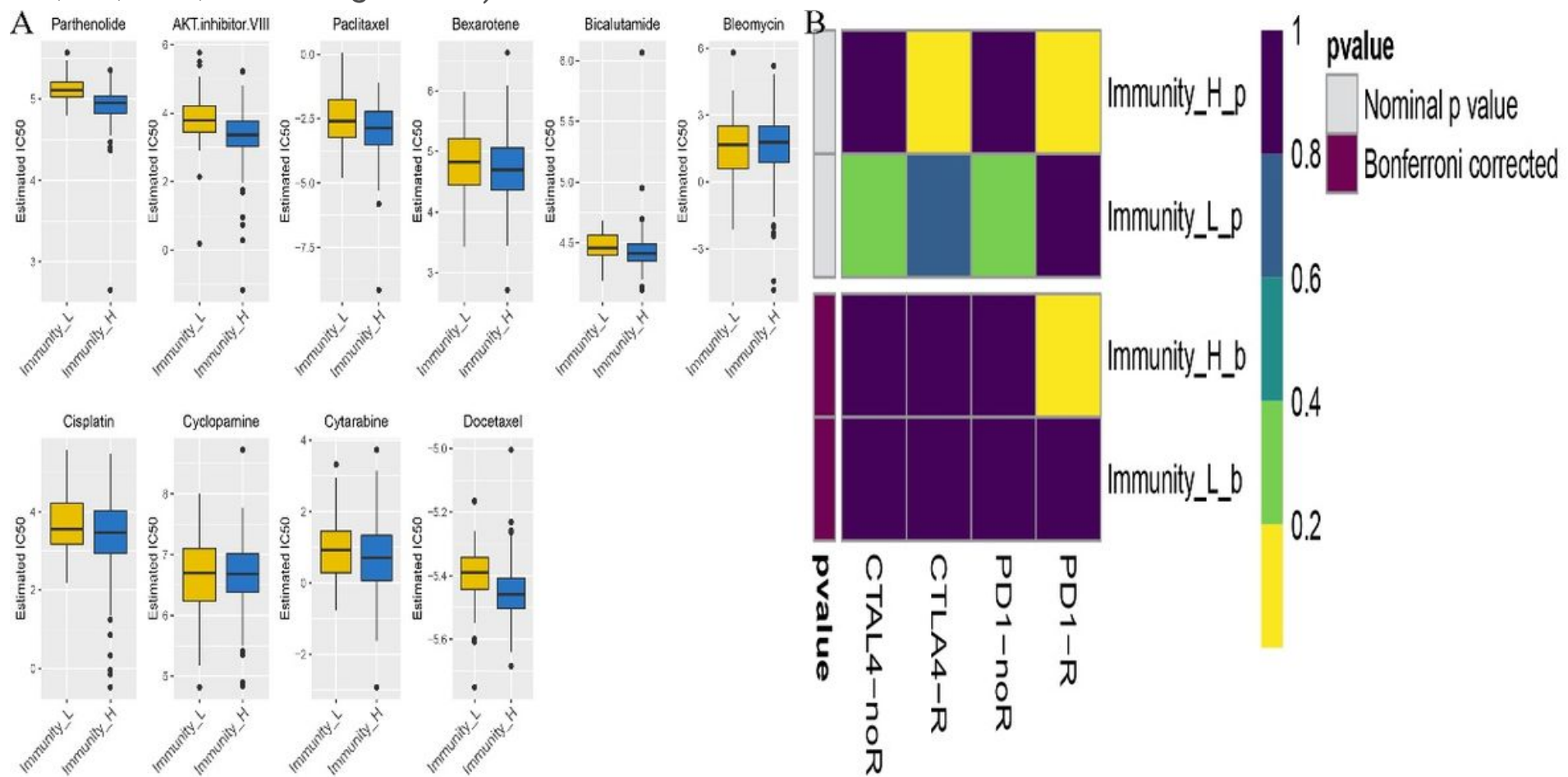

Figure 10 
Prediction for Immunotherapy and Anti-cancer Drug Response. The box plots show the estimated IC50 for chemotherapeutic drugs using the GDSC database: (A) for Immune-H and Immune-L OvCa subsets, (B) Submap algorithm demonstrated that Immune-H is more sensitive to the immunotherapy (Bonferronicorrected $\mathrm{P}<0.05)$.

\section{Supplementary Files}

This is a list of supplementary files associated with this preprint. Click to download.

- SupplementaryTable.pdf 